\title{
Peering into the dark (ages) with low-frequency space interferometers
}

\section{Using the $21-\mathrm{cm}$ signal of neutral hydrogen from the infant universe to probe fundamental (Astro)physics}

\author{
Léon V. E. Koopmans ${ }^{1}$ (D) - Rennan Barkana ${ }^{2}$ - Mark Bentum ${ }^{3,4}$. \\ Gianni Bernardi ${ }^{5,6}$ - Albert-Jan Boonstra ${ }^{4}$. Judd Bowman ${ }^{7}$. Jack Burns ${ }^{8}$. \\ Xuelei Chen ${ }^{9}$ - Abhirup Datta ${ }^{10}$. Heino Falcke ${ }^{11}$ - Anastasia Fialkov ${ }^{12}$. \\ Bharat Gehlot $^{7}$ • Leonid Gurvits ${ }^{13}$. Vibor Jelić $^{14}$ - Marc Klein-Wolt ${ }^{11}$. \\ Joseph Lazio ${ }^{15}$. Daan Meerburg ${ }^{16}$. Garrelt Mellema $^{17}$. Florent Mertens ${ }^{1,18}$. \\ Andrei Mesinger ${ }^{19}$. André Offringa ${ }^{4}$. Jonathan Pritchard ${ }^{20} \cdot$ Benoit Semelin $^{18}$. \\ Ravi Subrahmanyan ${ }^{21}$. Joseph Silk ${ }^{22,23}$. Cathryn Trott ${ }^{24}$. Harish Vedantham ${ }^{4}$. \\ Licia Verde ${ }^{25}$. Saleem Zaroubi ${ }^{1,26}$. Philippe Zarka ${ }^{18}$
}

Received: 29 January 2021 / Accepted: 18 March 2021 / Published online: 3 September 2021

(C) The Author(s) 2021

\begin{abstract}
The Dark Ages and Cosmic Dawn are largely unexplored windows on the infant Universe ( $z \sim 200-10)$. Observations of the redshifted 21-cm line of neutral hydrogen can provide valuable new insight into fundamental physics and astrophysics during these eras that no other probe can provide, and drives the design of many future ground-based instruments such as the Square Kilometre Array (SKA) and the Hydrogen Epoch of Reionization Array (HERA). We review progress in the field of highredshift 21-cm Cosmology, in particular focussing on what questions can be addressed by probing the Dark Ages at $z>30$. We conclude that only a space- or lunar-based radio telescope, shielded from the Earth's radio-frequency interference (RFI) signals and its ionosphere, enable the 21-cm signal from the Dark Ages to be detected. We suggest a generic mission design concept, CoDEX, that will enable this in the coming decades.
\end{abstract}

Keywords 21-cm cosmology · Dark ages $\cdot$ Cosmic dawn $\cdot$ Epoch of reionization · Space or lunar-based radio telescopes

Léon V. E. Koopmans

koopmans@ astro.rug.nl

Extended author information available on the last page of the article 


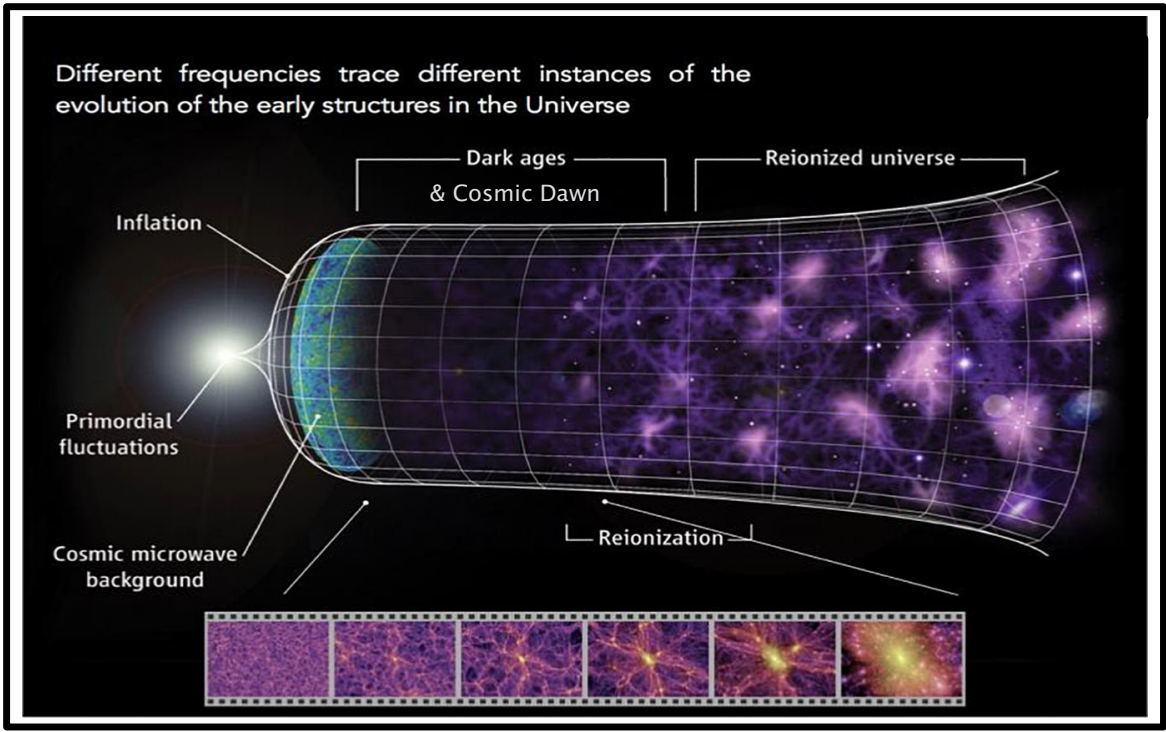

\section{Introduction}

The first billion years of the Universe (its 'infancy') is one of the least explored and understood eras in the Universe, despite enormous observational progress in recent years with optical/uv, infrared, and sub-mm ground- and space-based instruments. However, between the first image of the Universe from the Cosmic Microwave Background (CMB) and the first stars and galaxies becoming visible (some half to one billion years later) to large ground- and space-based observatories, observational probes remain incredibly scarce. Redshifts between $z \sim 11-1100$ are currently devoid of any direct observational probes. Even JWST in the 2020s will only be able to push direct observations a little further in redshift $(\mathrm{z} \sim 15)$ and only for a limited number of bright sources and over a limited field of view.

Neutral hydrogen, on the other hand, pervades the infant Universe and its redshifted 21 -cm signal allows one to chart the Universe from $\sim 10 \mathrm{kpc}$ to $\sim 100 \mathrm{Mpc}$ scales, via absorption and emission against the $\mathrm{CMB}$ and, potentially, via high-z radio sources. This signal allows one to probe astrophysical processes such as the formation of the first stars, galaxies, (super)massive black holes, and enrichment of the pristine gas from $\mathrm{z} \sim 6$ to $\mathrm{z} \sim 30$, as well as fundamental physics related to gravity, dark matter, dark energy and particle physics at redshifts beyond that. As one enters the so-called Dark Ages $(z>30)$, the Universe becomes pristine. Density perturbations are linear, and its physics is thought to be well understood. Deviations from linear-theory predictions of the 21-cm signal would thus usher new physics that is nearly impossible to probe at lower redshift where signals mix with those from complex astrophysical processes.

Ground-based low-frequency radio telescopes - e.g. LOFAR, MWA, OVROLWA, and NenuFar and the planned HERA and SKA - aim to detect the spatial fluctuations of the 21-cm signal. Complementary, global 21-cm experiments - e.g. EDGES (recently claiming a 21-cm signal detection at $\mathrm{z} \sim 17$ ), and SARAS - aim to measure the sky-averaged $21-\mathrm{cm}$ signal. All these instruments focus on redshifts $\mathrm{z} \sim 6$ 
to $\sim 25$ though. Pushing these observations to higher redshifts from the ground is impossible due to radio-frequency interference (RFI) and the ionosphere, even with the most powerful and isolated next-generation instruments such as SKA and HERA. New space-based radio-interferometers are critical in 2030+ to push observations of the spatially-varying 21-cm signal beyond $z \sim 25$.

Escaping RFI and the ionosphere has motivated space-based missions, such as the Dutch-Chinese NCLE instrument (currently in lunar L2), which is part of the Chinese Chang-e'4 mission; the proposed and partially funded US-driven lunar or space-based instruments DAPPER and FARSIDE; the lunar-orbit interferometer DSL (China); and PRATUSH (India), all missions to probe the global average or spatial fluctuations (FARSIDE, DSL) of the 21-cm signal. ESA is currently not involved in any of these pioneering missions, despite the tremendous discovery space that it will open and the promise for uncovering new (astro)physics. The importance of this era is emphasized by the numerous related White Papers submitted to the 2020 US Decadal Review, involving many researchers from the global community.

Some Key Questions that low-frequency space-based radio interferometers sensitive enough to probe the 21-cm signal from the Dark Ages $(\mathbf{z}>\mathbf{3 0})$ - will be able to probe, are related to: (i) inflation, (ii) the nature of dark matter and dark energy, (iii) gravity on scales currently inaccessible, (iv) particle physics (e.g. WIMPS, neutrinos, axions), (v) gravitational waves, (vi) non-gaussianity and (vii) primordial black holes. The same 21-cm signal observations at somewhat lower redshifts $(\mathrm{z} \sim 30-10)$ can also shed light, via tomography (impossible even with SKA or HERA), on never before seen astrophysical processes during the Cosmic Dawn, such as signatures of the formation of the first star, galaxies, stellar-remnant black holes, and the synthesis of elements heavier than helium.

To push beyond the current $\mathrm{z} \sim 25$ frontier, though, and measure both the global and spatial fluctuations (power-spectra/tomography) of the 21-cm signal, low-frequency (1$100 \mathrm{MHz}$; Bandwidth 50 MHz; z> 13) space-based interferometers with vast scalable collecting areas (1-10-100 $\left.\mathrm{km}^{2}\right)$, large filling factors $(\sim 1)$ and large fields-of-view $(4 \pi$ sr.) are needed over a mission lifetime of more than five years. In this ESA White Paper, we argue for the development of new technologies enabling interferometers to be deployed, in space (e.g. Earth-Sun L2) or in the lunar vicinity (e.g. surface, orbit, or Earth-Moon L2), to target this 21-cm signal. This places them in a stable environment beyond the reach of most RFI from Earth and its ionospheric corruptions, enabling them to probe the Dark Ages as well as the Cosmic Dawn, and allowing one to investigate new (astro)physics that is inaccessible in any other way in the coming decades.

\section{The first billion years of the universe}

Over the last hundred years, astronomers have discovered that we live in an everexpanding Universe which started 13.8 billion years ago in a very hot and dense state, commonly known as the Big Bang. The model which provides an excellent description of both the earliest phases and the more recent expansion is based on General Relativity and postulates that apart from ordinary, baryonic, matter, the Universe also contains a substantial amount of Dark Matter and Dark Energy. This model is known as ' $\Lambda$-Cold 
Dark Matter ( $\Lambda C D M)$ ' because of the specific properties of the Dark Matter (cold) and the Dark Energy (a constant $\Lambda$ ). In this model, the first hundreds of millions of years are a period in which the Universe, due to its expansion, became globally more and more rarefied and cold. At the age of about 380,000 years, the temperature dropped to about $3000 \mathrm{~K}$ and collisions were no longer able to keep the electrons from combining with the (mostly hydrogen) nuclei. As the Universe became neutral, baryonic matter decoupled from radiation, leaving the latter free to travel and allowing the former to follow the gravitational pull of tiny dark matter concentrations [110]. Over time, these local concentrations of dark and baryonic matter grew large enough to allow the formation of the first stars, called the Cosmic Dawn $(\mathrm{z} \sim 10-30)$. The era after decoupling and before the first stars $(\mathrm{z}>30)$ formed is commonly known as the Dark Ages and is the main focus of this White Paper.

\subsection{Probing the dark ages (and beyond) via the redshifted $21-\mathrm{cm}$ signal of neutral hydrogen}

The 21-cm hyperfine line of neutral hydrogen - central to the science in this White Paper - arises from the magnetic interaction between the proton and electron spins in neutral hydrogen. A spin-flip from the state with spins aligned to spins anti-aligned will emit a photon with a wavelength of $21.1 \mathrm{~cm}$ or a frequency of $1420 \mathrm{MHz}$. The connection of the atomic physics to astrophysics can be quite subtle and is an active area of research (see [49, 110], for reviews). For our purpose, we care about the emission or absorption of 21-cm wavelength light from hydrogen gas seen against some background source at some point in the cosmic time (called the '21-cm signal' hereafter). The intensity of the 21-cm signal depends upon the degree of ionization, density, temperature, and excitation state of the hydrogen gas in the cloud. Following the convention of radio astronomers, who describe the observed intensity of light in terms of a brightness temperature $\left(\mathrm{I}_{v}=2 \mathrm{kT} \mathrm{T}_{\mathrm{b}} v^{2} / \mathrm{c}^{2}\right)$, one can write the differential brightness temperature for the $21-\mathrm{cm}$ signal seen against some backlight as [110]:

$$
\delta T_{b} \propto \rho_{H I}\left(\frac{T_{S}-T_{\text {back }}}{T_{S}}\right)\left(\partial_{r} v_{r}\right)^{-1}
$$

Here $\rho_{\mathrm{HI}}$ is the density of neutral hydrogen and $\mathrm{T}_{\text {back }}$ describes the intensity of background radiation. $\partial_{\mathrm{r}} \mathrm{v}_{\mathrm{r}}$ is the local radial velocity gradient that controls the mapping between local and observed wavelengths - typically this will be determined by the cosmic expansion with minor corrections due to local peculiar velocities. $\mathrm{T}_{\mathrm{S}}$ is a spin temperature that describes the ratio of atoms in the spin-aligned versus spin-anti aligned states $\left(\mathrm{n}_{\uparrow \uparrow} / \mathrm{n}_{\uparrow \downarrow}=3 \exp \left[-\mathrm{h} v_{21} / \mathrm{kT}_{\mathrm{S}}\right]\right)$. The spin temperature is a key factor in interpreting the 21-cm signal $[6,107]$ and varies between the temperature of the CMB and the gas. At the earliest times (e.g. during the Dark Ages), it is set through thermal collisions between neutral hydrogen and other particles, which locks $\mathrm{T}_{\mathrm{S}}=\mathrm{T}_{\text {gas }}[1,142]$. As cosmic expansion dilutes the number density of hydrogen atoms, collisions become rare and the scattering of CMB photons relaxes $\mathrm{T}_{\mathrm{S}}=\mathrm{T}_{\mathrm{CMB}}$. Finally, as stars begin to illuminate the Universe (during the Cosmic Dawn) with their light, a process of 
resonant scattering of Lyman alpha photons, known as the Wouthuysen-Field effect, drives $T_{S}=T_{\text {gas }}[47,133]$. Since the gas temperature varies significantly over cosmic time, the $21-\mathrm{cm}$ signal can be viewed as a thermometer to measure the impact of cooling due to cosmic expansion or heating due to X-rays from stellar sources [107] or exotic physics, e.g. dark matter decay ([50]; see also below). We typically look for the $21-\mathrm{cm}$ signal as a spectral distortion of emission or absorption against some background radio source. For a very bright radio source, such as a radio quasar, the $21-\mathrm{cm}$ signal will always be seen as a series of absorption lines - the 21-cm forest [49]. Most generally for mapping the Cosmic Dawn or the Epoch of Reionization (EoR; see Fig. 1), where the $\mathrm{CMB}$ itself is the backlight, the 21-cm line can be seen either in absorption (when $\mathrm{T}_{\mathrm{S}}<\mathrm{T}_{\mathrm{CMB}}$ ) or emission $\left(\mathrm{T}_{\mathrm{S}}>\mathrm{T}_{\mathrm{CMB}}\right.$ ) depending upon the gas temperature and the degree of coupling. The $21-\mathrm{cm}$ signal is therefore extremely rich in information about the early Universe and its interpretation depends upon our ability to model the physical properties of hydrogen gas and the impact of radiation - Lyman alpha, ionizing or heating - from the first luminous sources [80, 108] during the Cosmic Dawn and after. Before the first sources emit radiation, however, astrophysics was physics. The Dark Ages are the main focus of this White Paper.
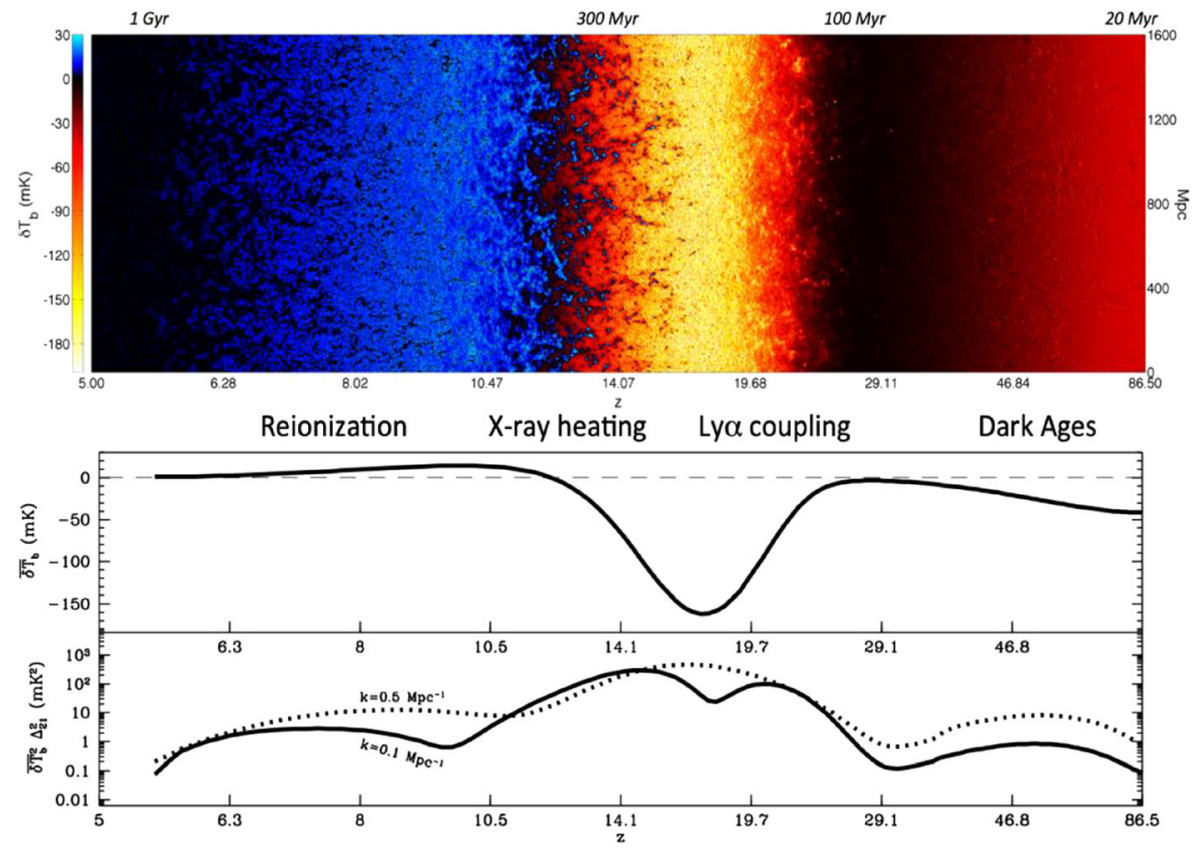

Fig. 1 2D slice through a mock 21-cm lightcone (top), corresponding to the global-signal evolution (middle), and the 3D power spectra at two wave numbers (bottom). Some major cosmic milestones can be seen from right to left: (i) Wouthuysen-Field (or Lyman- $\alpha$ ) coupling $[47,133]$, when Lyman-band photons efficiently couple the spin temperature to the gas temperature (black to yellow); (ii) epoch of heating, when galactic Xrays with long mean free paths heat the predominantly neutral IGM (yellow to blue); (iii) epoch of Reionization, when ionising photons drive the final major phase transition of our Universe (blue to black). The timing and the patterns of the signal encode a wealth of information about the first galaxies. This figure corresponds to the fiducial model of Mesinger et al. [81], though recent studies calibrated to high-z galaxy observations suggest that the Cosmic Dawn epochs of WF coupling and X-ray heating might occur later, with significant overlap with the EoR, due to less efficient star formation (e.g. [95]) 


\section{Cosmic eras in the infant universe: From physics to astrophysics}

Below, we focus on the Dark Ages and early Cosmic Dawn - which are both extremely hard to observe from Earth - and on the (astro)physical processes that can be probed using the 21-cm signal. We do not discuss the Epoch of Reionization (Fig. $1 ; z<10$ ) further, since it is expected to be fully characterized in the 2020 s by current and forthcoming ground-based instruments, and does not require space-based missions.

1. Dark Ages: The 21-cm signal of neutral hydrogen from the Dark Ages (DA) - a period of cosmic history preceding star formation during the Cosmic Dawn - is predicted to be a unique probe of cosmology and fundamental physics. A measurement of the $21-\mathrm{cm}$ signal from this era is expected to reveal unprecedented details about the early Universe, such as precision measurements of the scale dependence of primordial fluctuations (e.g. [78]), their possible deviations from Gaussian statistics (e.g. [77]) and even probe primordial gravitational waves through tidal effects and curl lensing [2, 13, 57, 73, 116, 119]. Furthermore, the Dark Ages signal will allow testing of fundamental theories in a completely unexplored regime. The shape of this signal depends on the underlying cosmological model (expansion history), nature of dark matter and the radio background radiation. Assuming the standard cold dark matter cosmology, conventional expansion history of the Universe (e.g., [103]) and the CMB as a background light, the 21-cm signal can be calculated precisely. Any deviations from the predicted signal could be a manifestation of exotic physics. For instance, extra radio background light in addition to the CMB would completely alter the shape of both the sky-averaged (global) signal and the power spectrum in a model-dependent way (e.g., [45]). Such deviations could be contributed to by neutrinos [25], dark matter decay [51, 106], or by superconducting strings [17]. The Dark Ages 21-cm signal will also be an important probe of dark matter physics (e.g., [123-126]) allowing one to test different particle physics models of dark matter in currently unconstrained regimes. For example, the annihilation rate of Weakly Interacting Massive Particles (WIMPs) is expected to be higher in the dense environment of the early Universe than today (e.g. [24]). By-products of dark matter annihilation (or decay) will heat and ionize the gas imprinting characteristic signature in the $21-\mathrm{cm}$ signal. In another class of models where dark matter directly interacts with baryons (e.g., [126]), thermal history of the gas is also modified. A smoking gun signature of interacting dark matter is in the $21-\mathrm{cm}$ power spectrum where, if the interaction cross-section is velocity-dependent, enhanced Baryon Acoustic Oscillations are expected (e.g., [44, 88]). Finally, models in which dark matter is warm or have a large coherence scale (e.g., ultra-light axions, sterile neutrino) predict delayed star formation and leads to an extended Dark Ages.

2. Cosmic Dawn: The Cosmic Dawn (CD) of the first galaxies heralded the end of the Dark Ages and the birth of structure. Baryons cooled onto dark matter halos, collapsing to form the first stars, black holes, and galaxies. Their light spread throughout the Universe, eventually heating and ionizing virtually every baryon. The CD culminated with the Epoch of Reionization (EoR), whose timing we have recently begun to nail down thanks to observations of the CMB (e.g. [102]), highredshift QSOs (e.g. [5, 87]) and galaxies (e.g. [72]). However, the CD should 
contain much more information than just the EoR. Ionizing photons responsible for the EoR have relatively short mean free paths through the intergalactic medium (IGM) of order kpc. However, soft UV and X-ray photons from the first galaxies can travel distances of $\sim 100 \mathrm{Mpc}$. These radiation fields permeate the Universe while the Cosmic HII regions from the EoR are still proximate to the nascent galaxies. Empirical scaling relations of the soft UV and X-ray luminosities to star formation rates (SFRs) of local galaxies (e.g. [18, 69, 82]) suggest that the soft UV and X-ray backgrounds established during the CD are sufficiently strong to couple the spin temperature of the cosmic gas to its kinetic temperature (allowing us to see the cold gas in contrast against the $\mathrm{CMB}$ ), and subsequently heat the gas to temperatures above the $\mathrm{CMB}([75,93]$; see Fig. 1$)$. Indeed the $C D$ is expected to result in the strongest $21-\mathrm{cm}$ signal, with the adiabatically-cooling IGM providing a large contrast to the hot IGM proximate to the first X-ray sources (Fig. 1). What can we learn from CD observations? The timing and spatial structure of the cosmic 21-cm signal is sensitive to the nature of the sources. The very first galaxies which start the CD are likely hosted by so-called mini-halos (with masses of $10^{6-8} \mathrm{M}_{\odot}$ ) accreting their gas through $\mathrm{H}_{2}$ cooling (e.g. [55]). Mini-halos likely have very different interstellar medium (ISM) properties and star formation efficiencies compared to later galaxies (e.g. [63, 135]). Due to their relatively pristine gas reservoir, they likely host stars which were more massive and brighter, so-called Population III stars $[115,128,138]$. Although mini-halo galaxies likely start the $\mathrm{CD}$, star formation inside them is soon sterilized with the buildup of an $\mathrm{H}_{2}$ dissociative background (e.g. [42, 59]). Atomic hydrogen subsequently provided the dominant cooling mechanism for galaxies inside $>\sim 10^{8} \mathrm{M}_{\odot}$ halos. Such dwarf galaxies are still far too faint to be directly observed. However, we hope to be able to extrapolate some of their properties from the brighter high-z galaxies to be observed with JWST (e.g. [118]). These first generations of galaxies host massive stars, whose soft UV radiation couples the spin temperature to the gas temperature, allowing us to see the cold IGM against the CMB. The timing of this WF coupling tells us when these first stars appeared and disappeared, what was their typical initial mass function (IMF), and how efficient was star formation. The stars in the first galaxies also are responsible for heating the IGM, likely through high-mass Xray binaries (HMXBs) and the hot ISM created following supernovae events (e.g. [76]). The X-rays from HMXBs and the hot ISM can permeate deep into the neutral IGM, well before the bulk of the EoR, partially ionizing it and heating it. The spatial structure of this epoch of heating $(\mathrm{EoH})$ is also a sensitive probe of the nature of the dominant X-ray sources (e.g. [43, 93]), as well as the ISM properties of the host galaxies which attenuate the emerging soft X-rays (e.g. [35]). Similarly to the DA, the CD could also hold clues about even more exotic radiation sources. If dark matter annihilation is an efficient process, the resulting shower could provide a fairly uniform background of X-rays, whose spatial signature is dramatically different to that of astrophysical sources (e.g. [36, 70, 129]). Moreover, if the recent, putative EDGES detection of an absorption signature at $\mathrm{z} \sim 17$ is proven to be cosmological [15]; see however [16, 31, 56]), the spatial structure of this epoch could tell us either about a population of radio loud mini QSOs [39] or an exotic interaction between DM and baryons [7, 89]. Indeed, the later mechanism could allow us to more easily measure baryonic acoustic oscillations at redshifts of $\mathrm{z} \sim 20$ 
$[75,90]$. In summary, the timing and structure of the Cosmic Dawn 21-cm signal will result in an invaluable physical bounty. By mapping the Cosmic Dawn, we will be able to understand the nature of the first stars, galaxies, (super-massive) black holes, the stellar and ISM properties, and possibly even dark matter.

Global signal versus spatial intensity fluctuations Complementary aspects of the $21-\mathrm{cm}$ signal can be studied via two different approaches. The sky-averaged or global signal (monopole in CMB parlance) can be measured by a spectrometer connected to a single dipole antenna (see Fig. 1, bottom panel and Sections 5 \& 6). Alternatively, angular fluctuations in the $21-\mathrm{cm}$ brightness can be studied at different frequencies via interferometric techniques (see Fig. 1, top panel and Sections $5 \&$ 6). The global and spatially fluctuating signals are analogous to the diffuse $2.7 \mathrm{~K}$ blackbody and anisotropies seen in the $\mathrm{CMB}$ respectively. Unlike the $\mathrm{CMB}$, the $21-\mathrm{cm}$ signal, being a spectral line, allows for line-of-sight tomography of the IGM. The measured 21-cm differential brightness temperature (Eq. 1) primarily depends on the radiation temperature, gas temperature and gas ionization fraction (assumed zero during the CD/DA). In the absence of stellar sources of radiation in the Dark Ages, all three quantities are strongly constrained by standard baryonic physics applied to an adiabatically expanding Universe permeated by CMB photons. Any departure from theoretical expectations of the global radio spectrum in the Dark Ages (Fig. 1) requires an exotic mechanism to substantially alter the physical condition of baryonic matter, and/or the cosmic radiation background on cosmic scales (see above). An accurate global radio spectrum measured by a single dipole (Section $5 \& 6$ ) can therefore reveal the presence of a new mechanism to deposit energy into gas on cosmological scales. The predictions are similarly strong for the spatial fluctuations of the 21-cm signal from the Dark Ages, which in the absence of luminous sources (no non-linear structure formation and baryonic feedback), are largely governed by linear structure formation. The resulting baryonic density fluctuations and peculiar velocity have been computed for the standard CDM paradigm (Section 7) and yield relatively stringent predictions for the power spectrum of the 21-cm signal that interferometry can measure. Measured departures will therefore significantly evolve our understanding of the nature of dark matter and its influence on structure formation. We discuss both approaches in Section 6 and 7, although the focus in Section 7 will be on future (in 2030+) interferometric 21-cm signal observations of the Dark Ages and early Cosmic Dawn.

\section{General motivation and selected key science drivers for $21-\mathrm{cm}$ signal observations}

The Cosmic eras discussed above constitute the transitional steps that take our Universe from its early linear phases to the highly non-linear Universe that we currently see around us. Each of these eras ushers the Universe into a new realm, and hence probes different physical processes that can be used to understand the onset of structure formation in the Universe as well as the very nature of the initial conditions in the Universe. The redshifted $21-\mathrm{cm}$ line is uniquely suited to study these phases of the Universe's evolution for a number of reasons. Firstly, it probes the bulk of the 
hydrogen in the IGM and provides us with a global picture of the physical state of the Universe. Secondly, since it comes from a specific wavelength, it allows a detailed tomographic reconstruction of the Universe's history as a function of redshift. Thirdly, it is one of the very few observables (if not the only one) that are accessible to us in this redshift range, either from the ground or, for the longer wavelengths, from space. During the Dark Ages of the Universe the 21-cm line allows us to detect the intergalactic medium in absorption against the $\mathrm{CMB}[58,71,117]$. Since in this stage the matter fluctuations in the Universe are still mostly well within the linear regime of gravitational instability, they faithfully reflect the initial conditions of the Universe in a very detailed manner. Hence, the redshifted $21-\mathrm{cm}$ can be used to e.g. constrain models of inflation or its alternatives, through the study of non-Gaussianity with high order statistics (see Section 3 for more details and references). Such data promises to be even more constraining than the CMB since it covers a wide range of redshifts. The 21-cm line from this era can also be used to study many other topics, e.g., extreme density peaks (as progenitors of supermassive black holes), annihilating and decaying dark matter particle candidates, etc. During the Cosmic Dawn phase the first objects are expected to form and allow us to study the interplay between the Lyman-alpha coupling to the cooler baryons and of the spin temperature $[46,47,133]$ and X-ray heating of the IGM [71, 109]. During this era the IGM is still mostly neutral but the first stars and Xray sources leave a marked imprint on the fluctuations of the spin temperature in the IGM, most significantly on the contrast between absorption and emission regions of the $21-\mathrm{cm}$ signal. The structure and evolution of this spectrum of negative and positive fluctuation shed light on the processes that formed the first objects, when they formed, and how. This is also the period in which the supermassive black holes that we detect almost routinely now between redshift 6 and 7 formed [40, 41, 87]. Therefore exploring this era might answer the puzzle of supermassive black hole formation and the rapid build up of their mass in such a short period in the early history of the Universe. This period might also reveal to us new physics as the result from the EDGES telescope tentatively suggest [15]. Of course, also here there is a great potential to address cosmological questions. The last of these phases is the Epoch of Reionization (Fig. 1), which is targeted by many current telescopes. The 21-cm signal from this epoch informs us about the onset of the Reionization process, and how long it took to permeate the whole intergalactic medium to fill the Universe (see e.g., [139]). The answer to the questions that arise in the context of studying the Dark Ages and Cosmic Dawn (and the Epoch of Reionization) relate to many fundamental issues in cosmology, galaxy and quasar formation, and the physics of very metal poor stars; all at the heart of many research issues in modern astrophysics (Fig. 2).

\subsection{Some key science drivers to observe the dark ages}

Tomography of the 21-cm signal at observed frequencies of $\sim 1$ to $\sim 100 \mathrm{MHz}$ thus provides access to a previously inaccessible volume of parameter space and samples a very large number of independent modes describing the primordial fluctuation field. This will open a new window on information content about the properties of the early Universe, and is highly complementary to that embedded in the CMB temperature and polarisation signals. 
Key research topics addressed via the $21-\mathrm{cm}$ signal

\section{Dark Ages (z 30-200)}

- Physics of gravity

- Gravitational waves

- Dark Matter \& Dark Energy

- Particle physics (e.g. WIMPs, axions, neutrinos)

- Primordial black holes

- Inflationary physics

- Non-Gaussianity

- Baryon-Dark-matter interactions

- ...

\section{Cosmic Dawn}

( $z \sim 10-30)$

- First stars (Pop III/II)

- Formation of first galaxies

- Stellar remnants/HMXRBs

- Seeds of SMBHs

- Synthesis of metals and enrichment of the IGM

- Molecular cooling

- ...

Fig. 2 Some of the key questions that the 21-cm signal from the Dark Ages and the Cosmic Dawn can address

- $\mathbf{f}_{\mathrm{NL}}$, single vs multi-field inflation: Because of the stringent Planck limits in the $\mathrm{r}-$ $\mathrm{n}_{\mathrm{s}}$ plane, viable inflationary models today mostly have very low $\mathrm{r}$. This means that CMB polarisation experiments need exquisite sensitivity and wide sky coverage to qualitatively improve our understanding of inflation. However, there is no guaranteed prediction of $r$. Primordial non-gaussianity provides a robust but challenging complementary probe of inflation, even the simplest inflationary models predict small but non-zero deviations from gaussianity, while most multi-field inflation models are expected to have $\mathrm{f}_{\mathrm{NL}} \sim 1$ and many predict features in $\mathrm{f}_{\mathrm{NL}}$. The errors bars on $f_{\mathrm{NL}}$ scale with the (inverse of the) number of independent modes. The limited number of modes in the CMB $\left(\sim 10^{6}\right)$ and in large-scale galaxy surveys $\left(\sim 10^{8}\right)$ strongly motivates exploration of 21-cm Dark Ages cosmology at $\mathrm{z} \sim 25-75$ (20-60 MHz) where up to $10^{12}$ modes can be explored. Attainment of a robust limit on (or detection of) primordial non-gaussianity would provide the ultimate probe of generic inflation.

- Primordial black holes: Primordial black holes (PBHs) are the only dark-matter candidate that avoids introducing new beyond-standard-model physics and are motivated by gravitational wave observations, but the remaining window for $\mathrm{PBH}$ to be a significant DM contributor is limited to the sub-lunar and to the solar-mass range. One needs boosted scale-dependent and possibly non-Gaussian conditions, available in many and possibly all inflationary models. These may lead to spectral power boosts, once accretion and Poisson clustering are included, as well as to IGM heating, that can be uniquely probed via very low frequency $21-\mathrm{cm}$ Dark Ages observations, during an era well before any astrophysical sources have formed that complicate interpretations. 
- Dark-matter annihilation and decay: Identification of a weakly interacting darkmatter particle candidate is the holy grail of particle astrophysics. The Dark Ages offer a unique forum for exploring long-lived particle annihilations or decays because the $21-\mathrm{cm}$ signal and especially its fluctuations are highly sensitive to electromagnetic energy injection. This allows study of dark-matter models in a complementary way to direct detection, via precision spin temperature measurements, that should provide order-of-magnitude improvements over the best current Dark-Ages limits from CMB constraints. The advantage of measuring DM annihilations and decays during the Dark Ages is that it provides a direct probe of exotic physics, uncontaminated by astrophysical processes.

- Ultra-light Axions: The axion is a hypothetical dark-matter particle that would explain the lack of charge-parity violation in strong interactions, and that naturally emerges from string theory. It represents an interesting dark-matter candidate and many experimental searches are being planned. Moreover, the ultralight axion uniquely combines quantum and astronomical scales, with its de Broglie wavelength potentially measurable via the ultra-light axion's (ULX) mass, and potentially mitigates small-scale observational tensions within the $\Lambda$ CDM paradigm. Ultra-light axion signatures on the matter power spectrum appear on very small scales, making observations of 21-cm absorption against the CMB during the Dark Ages a unique observable to set competitive constraints on its mass, complementing and potentially surpassing CMB probes.

- Supermassive Black Holes: There is still no convincing scenario for the origin of the seeds of supermassive black holes that are located in the centres of most massive galaxies. If these are seeded by a population of primordial black holes, gas accretion around PBHs during the cosmic dark ages would leave a unique signature in the $21-\mathrm{cm}$ signal. The presence of a population of primordial black holes would change the standard ( $\Lambda$ CDM-based) interpretation of the CMB anisotropy signal, opening up degeneracies among cosmological parameters and relaxing current $\Lambda \mathrm{CDM}$-based constraints, hence providing complementarity of the two probes.

- DM-baryon relative velocity: Baryons and Cold Dark Matter have supersonic relative velocities after recombination. This relative velocity suppresses the growth of matter fluctuations, and the amplitude of the small-scale power spectrum is modulated on scales over which the relative velocity varies, and leaves an imprint in the small-scale, very high-z matter power spectrum. DM models that suppress the small scale spectrum (such as warm dark matter and ULAs) would cancel this modulation. Therefore, this observable can both test $\Lambda \mathrm{CDM}$ predictions and DM models.

\section{Current/planned ground-based observatories}

A wide range of low-frequency arrays have been either used, or specifically designed, to detect the redshifted $21-\mathrm{cm}$ signal of neutral hydrogen over the past decade (Fig. 3). Some have already been decommissioned, or are being upgraded. Among them are the 21CMA (China), GMRT (India), PAPER (US/South Africa), the latter no longer active, LOFAR (The Netherlands), MWA (Australia), and OVRO-LWA (US) instruments, 

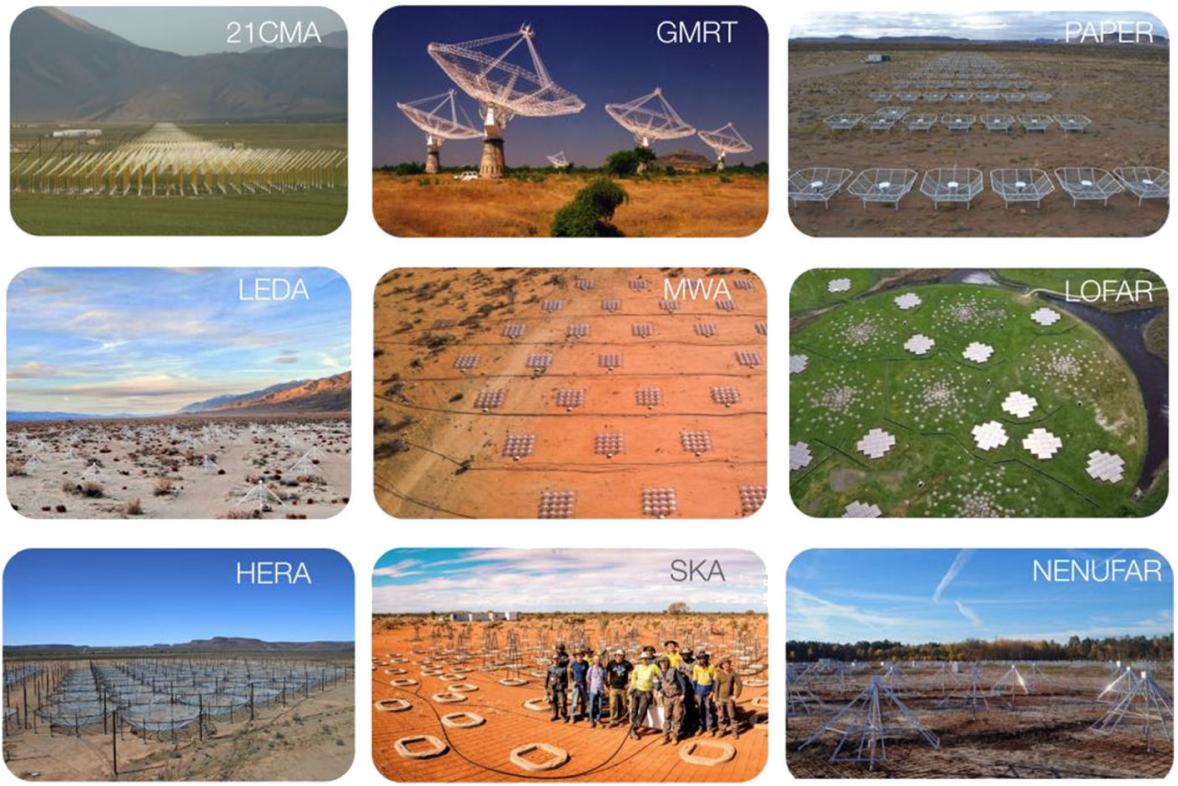

Fig. 3 Panel of the current and planned 21-cm signal experiments (PAPER is decommissioned), focussing largely on probing the Epoch of Reionization $(\mathrm{z} \sim 6-10)$ and late Cosmic Dawn $(\mathrm{z}<25)$, in no particular order. See text for details

and planned arrays such as HERA (South Africa) and the SKA (Australia and South Africa). All instruments have the Epoch of Reionization as their main target, since the signal-to-noise is expected to be largest, and least affected by the ionosphere and (extra)Galactic foregrounds, and RFI. Some target the Cosmic Dawn as well, but detections are only feasible in case of very large ('exotic' 21-cm signal; see above). Below, a short summary is provided for each of the instruments, each with their own specific design and technology, allowing one to set the scene for future space-based instruments.

21CMA ${ }^{1}$ The 21 Centi-Meter Array (e.g. [141]) is situated in the Tian Shan Mountains of western China. It is a meter-wave interferometric array designed to probe the $21-\mathrm{cm}$ signal from the Cosmic Dawn and the Epoch of Reionization at $z=6-27$. The array was constructed between August 2005 and July 2006 and upgraded by July 2010. It consists of 81 pods, each with 127 log-period antennas, which are deployed along two perpendicular arms of 6 and $4 \mathrm{~km}$, respectively. A field of 10-100 square degrees, centred on the North Celestial Pole (NCP), is imaged $24 \mathrm{~h}$ per day in the frequency range of 50-200 MHz and with a spectral resolution of $24 \mathrm{kHz}$. Coherent uv-data at each frequency channel are being accumulated to meet the desired sensitivity of a statistical detection of the 21-cm signal, and advanced RFI and foreground removal techniques have been developed.

GMRT $^{2}$ The Giant Metrewave Radio Telescope is a radio interferometer consisting of 30 antennas, each with a diameter of $45 \mathrm{~m}$. Fourteen of these are arranged in a $1-\mathrm{km}$ 
dense central core which allows high-brightness sensitivity required to search for the 21-cm signal [101]. The longest separation between antennas is about $25 \mathrm{~km}$. Data were taken over 5 nights in December 2007, accumulating about $40 \mathrm{~h}$. The observations were centred on PSR B0823 + 26, used to calibrate the instrument and ionosphere. The primary beam has a full width half-maximum (FWHM) of $3.1^{\circ}$ and a maximum angular resolution of about 20 arcsec. The bandwidth covers a frequency range from 139.3 to $156.0 \mathrm{MHz}$ in 64 frequency bins of $0.25 \mathrm{MHz}$ each with a time resolution of $64 \mathrm{~s}$. This corresponds to a redshift range of $\mathrm{z}=8.1-9.2$, providing an upper limit of $(248 \mathrm{mK})^{2}$ for $\mathrm{k}=0.50 \mathrm{~h} \mathrm{Mpc}^{-1}$ at $\mathrm{z}=8.6$ at the 2-sigma level [94].

Paper $^{3}$ The Precision Array to Probe the Epoch of Reionization was located in Karoo reserve area in South Africa. It was conceived to be a reconfigurable interferometric array to be deployed in stages of increasing number of elements (up to a maximum of 128 elements). It used individual $2 \mathrm{~m}$ dipoles sensitive to the 100-200 $\mathrm{MHz}$ range that were first deployed in pseudo random configurations to test calibratability and observe properties of foreground sources (e.g., $[64,96,104]$ ). The PAPER team spearheaded a novel approach to the detection of the $21-\mathrm{cm}$ signal by adopting highly redundant array configurations in order to maximize sensitivity on a number of power spectrum modes [97]. The drawback of redundant arrays is that they provide limited imaging capabilities and, therefore, limited foreground modelling capabilities. This led to the adoption of the so-called avoidance method: smooth-spectrum foregrounds are expected to be confined to a specific region of power spectrum space, leaving the remaining uncontaminated; this uncorrupted area is where the 21-cm signal detection should be attempted (e.g., [97]). Power spectrum results were published and currently revisited in an upcoming publication [66].

LOFAR $^{4}$ The Low-Frequency Array (LOFAR; van [54]) has the detection of the 21-cm signal of neutral hydrogen during the Epoch of Reionization (EoR; see, e.g. [98]) as one of its key-science drivers. LOFAR is also a pathfinder to the Square Kilometre Array (in particular SKA-low). The few-km inner core of LOFAR's High Band Antenna (HBA; 110-240 MHz) system consists of 48 stations each having 24 tiles of each $4 \times 4$ cross-dipoles. The large collecting area in the core and the high filling factor of LOFAR-HBA make it particularly suited for the detection of diffuse low-surface brightness emission of neutral hydrogen during the EoR. Besides LOFAR observing in standard station beam-forming mode, it can also cross-correlate all 576 HBA tiles or LBA dipoles (see below) of the inner 12 stations, yielding an extremely large field of view (e.g. [53]) and hence probe a larger volume of the Universe, reducing sample variance that dominate the largest scales of the $21-\mathrm{cm}$ signal. The Low-Band Antenna system (i.e. (10)-30 MHz) could, in principle, detect the 21-cm signal from the Cosmic Dawn (CD) as well (e.g. [52, 53]), but only if the 21-cm signal exceeds nominal predictions of $10-100 \mathrm{mk}^{2}$ at $\mathrm{k}=0.1 \mathrm{cMpc}^{-1}$ by a significant fraction (e.g. [44, 45]). The reason is the extremely bright Galactic foregrounds that cause a high noise level. The second reason is that ionospheric effects are far more significant at lower frequencies, causing signal distortions. Currently, LOFAR has yielded the deepest

\footnotetext{
${ }^{3}$ http://eor.berkeley.edu/

${ }^{4}$ http://www.lofar.org/
} 
upper limits on the $21-\mathrm{cm}$ signal from both the EoR (i.e. $(79 \mathrm{mK})^{\wedge} 2$ for $\mathrm{k}=0.06 \mathrm{~h}$ $\mathrm{Mpc}^{-1}$ at $\mathrm{z} \sim 10$; [98]) and the CD ( $\mathrm{z} \sim 25$; [52]). Observing the EDGES2 21-cm signal (see below) by cross-correlating all 576 dipoles of the inner 12 LBA stations (i.e. Amsterdam-ASTRON Radio Transients Facility And Analysis Center (AARTFAAC); [53]) is ongoing.

MWA $^{5}$ The Murchison Widefield Array $[127,132]$ is a low-frequency telescope in the Western Australian desert, on the same site of the future SKA. It comprises 256 tiles of 16 dual-polarisation dipoles in a regular $4 \times 4$ pattern, spread over $5 \mathrm{~km}$. Detection of the signal from the EoR is one of its primary science goals, and it contains two subarrays of 36 tiles in a redundant hexagonal pattern to maximize sensitivity to EoR spatial scales, and provide additional instrument calibratability. The MWA is pursuing a statistical detection of the spatial fluctuation power spectrum, and has auxiliary programs to provide early results from cross-correlation studies, and pursuit of alternative statistics. Its large, 25 degree field-of-view improves sample variance, but at the cost of substantial leakage of power from sources close to the horizon into the EoR dataset. Smooth bandpass calibration, wide-field foregrounds and adequate sky models remain the primary challenges for undertaking EoR science with the MWA. Nonetheless, the EoR collaboration has published competitive upper limits [9] to the power spectrum on 10 arcmin to 1 degree scales at $z=6.5-9$, and is also pursuing some analysis in the Cosmic Dawn at $\mathrm{z}=15-18$ (e.g. [37]).

OVRO-LWA ${ }^{6}$ The Owens Valley Radio Observatory Long-Wavelength Array (OVRO$L W A$ ) is located in Owens Valley, California. It operates in the $30-88 \mathrm{MHz}$ frequency range corresponding to $\mathrm{z} \sim 15-46$, seeking to detect the 21-cm signal from the Cosmic Dawn. It aims to observe both the global signal (the experiment LEDA, Large aperture Experiment to detect the Dark Ages) via (five) individual dipoles equipped with custom-built calibration sources and 21-cm fluctuations via an array of 256 dipoles. Dipoles are pseudo randomly distributed to achieve an essentially filled array within a $\sim 200 \mathrm{~m}$ diameter core, providing excellent imaging capabilities to Galactic diffuse emission - the brightest foreground component. The OVRO-LWA approach to measure the 21-cm signal can be versatile, allowing to image and subtract foregrounds but also to isolate them in the power spectrum domain without any specific modelling. Current simulations shows that if IGM heating occurs efficiently at $\mathrm{z} \sim 16$, OVRO-LWA would be able to detect the $21-\mathrm{cm}$ power spectrum at $\mathrm{k} \sim 0.1 \mathrm{Mpc}^{-1}$ with $\mathrm{a} \sim 10$ sigma signalto-noise ratio in $3000 \mathrm{~h}$. First observations have set a $10^{8}(\mathrm{mK})^{2}$ upper limit on the 21$\mathrm{cm}$ power spectrum at $\mathrm{k}=0.1 \mathrm{Mpc}^{-1}$ at $\mathrm{z}=18.4$ [32]. Limits on the global signal can, and have, also been set with outrigger dipoles (e.g. [4]).

NenuFar $^{7}$ New Extension in Nançay Upgrading LOFAR (NenuFAR; [140]), situated in Nançay (France), is an extension of LOFAR but also a standalone instrument in the low-frequency range (10-85 MHz). Antennae were modelled on the LWA design whereas preamplifiers were designed in France. Antennas are distributed in 96 mini-

\footnotetext{
${ }^{5} \mathrm{http} / / /$ www.mwatelescope.org/

${ }^{6} \mathrm{http} / / / \mathrm{www} . t a u c e t i . c a l t e c h . e d u / L W A / ;$ see also for LEDA: http://www.tauceti.caltech.edu/leda/

${ }^{7}$ https://nenufar.obs-nancay.fr/en/homepage-en/
} 
arrays ( $>56$ in place) each with 19 dual-polarised elements, densely covering a disc of $400 \mathrm{~m}$ in diameter, making it the most sensitive low-frequency array currently in operation. Several mini-arrays are situated at distances of 2-3 km. Receivers will include the LOFAR backend, a local beam-former, and a COBALT correlator. The NenuFAR concept has many points in common with GURT (the Giant Ukrainian Radio Telescope), with which it shares some technical studies, and its exploitation will benefit from a coordination with UTR-2. In the summer of 2019, a Key Science Programme started ( $>1000$ h of observations in 2019-2020) with NenuFar to observe the 21-cm signal from the Cosmic Dawn, in particular to confirm or reject predictions made from the EDGES feature [7, 44].

Hera ${ }^{8}$ The Hydrogen Epoch of Reionization Array (HERA) is an array currently under construction in the Karoo reserve area in South Africa. HERA is built following the approach used for PAPER: a highly redundant array to maximize the sensitivity on a number of power spectrum modes measured using the avoidance approach. In order to increase the sensitivity with respect to PAPER, it employs $14 \mathrm{~m}$ diameter dishes that, in the final configuration, will be densely packed in a highly redundant hexagonal array configuration of $\sim 350 \mathrm{~m}$ diameter. HERA is built with the purpose to provide a complete statistical characterization of cosmic reionization: its high brightness sensitivity configuration leads to a significant power spectrum detection in the $\mathrm{Mpc}^{-1}$ range throughout Reionization (i.e., [30, 105]), fully constraining the evolution of the IGM neutral hydrogen fraction. As the avoidance approach does not take advantage of foreground modelling, particular attention was paid to prevent the instrumental frequency response from corrupting intrinsically smooth foregrounds [38, 100]. HERA is currently under construction, with more than 200 dishes deployed and science observations routinely carried out [23, 65]. New feeds that extend the sensitivity to the 50-250 MHz (i.e. enabling observations of the Cosmic Dawn) are currently deployed for testing. In summary, HERA is planned to deliver a complete characterization of cosmic Reionization and to attempt the detection of the Cosmic Dawn. Given its redundant configuration, imaging capabilities remain limited and will be the target of a next generation experiment.

SKA $^{9}$ The low-band system (SKA1-Low) of the Square Kilometre Array (SKA), is a low-frequency aperture-array radio telescope system, made up of $\sim 131,000$ wide-band antennas, half of which will be situated in a high-filling factor dense core of $\sim 0.2 \mathrm{~km}^{2}$. It will operate from $50 \mathrm{MHz}$ to $350 \mathrm{MHz}$ and be located in Western Australia. SKA1low is particularly suitable for sensitive $21-\mathrm{cm}$ signal observations of both the Epoch of Reionization, where direct imaging can be done down to the $\mathrm{mK}$ level on $\sim 10$ arc minute scales, and of the Cosmic Dawn, where it is expected to yield detailed $21-\mathrm{cm}$ signal power-spectrum measurements up to redshifts $\mathrm{z} \sim 25[67,79]$. SKA1-Low (as HERA), despite its sensitivity and collecting area, however, remains unable to observe beyond $\mathrm{z} \sim 25$ because of its frequency cutoff at $50 \mathrm{MHz}$, motivated both by the presence of (strong) human-made RFI and very large errors induced in the data by the time-varying ionosphere.

\footnotetext{
${ }^{8}$ https://reionization.org/

${ }^{9} \mathrm{https}: / / \mathrm{www}$. skatelescope.org/See also the SKA science book at https://www.skatelescope.org/books/
} 
Whereas the above instruments aim to measure the spatial fluctuations of the $21-\mathrm{cm}$ signal, the instruments below aim to measure its global sky-averaged brightness temperature (see above). We briefly discuss EDGES and SARAS. Besides these two extremely precisely designed and leading mono/dipole instruments (with deepest limits or claimed detection), a range of other global 21-cm signal experiments are currently ongoing such as e.g. PRIZM, BIGHORNS and SKIHI, which we will not further discuss because they ceased operations or have not yet set competitive limits.

Edges $^{10}$ The U.S.-led Experiment to Detect the Global EoR Signature (EDGES) is located in Western Australia. Over the last decade, it has operated four instruments covering frequencies spanning the Cosmic Dawn and Reionization eras. Each instrument consists of a well-calibrated radio receiver connected to a single compact dipolelike antenna, chosen to minimize frequency-dependent effects. Building on techniques similar to those employed in other microwave measurements [10, 60], EDGES has demonstrated end-to-end absolute measurement calibration [83, 112] at the levels of accuracy needed for $21 \mathrm{~cm}$ observations. The experiment has disfavoured rapid Reionization $[14,84]$ and specific astrophysical models of early star formation, particularly those with little or late X-ray heating [85, 86]. In 2018, the EDGES team reported the first evidence for detection of the redshifted $21 \mathrm{~cm}$ signal. They found an absorption profile in the sky-averaged radio spectrum centered at a frequency of $78 \mathrm{MHz}$, corresponding to redshift 17, and with an amplitude of $0.5 \mathrm{~K}$ [15]. The amplitude of the observed profile is more than a factor of two greater than the largest standard predictions and suggests, surprisingly, that either the gas between early stars and galaxies was significantly colder than expected or that the background radiation temperature was hotter than expected. A number of possible explanations have been proposed, including interactions between baryons and various candidate dark matter particles (e.g. [7, 88]), as well as possible astronomical sources capable of producing an intense radio synchrotron background in the early Universe (e.g. [39]). Efforts are presently underway in the 21-cm community to confirm the EDGES detection (Figs. 4 and 5).

SARAS $^{11}$ At the Raman Research Institute in India, a progression of radiometers have been constructed and deployed using Shaped Antennas to measure the background RAdio Spectrum (SARAS); collectively referred to as the SARAS radiometers. The radiometers have optimized their efficiencies in the 50$200 \mathrm{MHz}$ band, with the goal of detecting global spectral distortions from redshifted 21-cm signals from Cosmic Dawn and Reionization. The SARAS antennas are all electrically small thus providing frequency independent beams and avoiding mode coupling of sky spatial structures into confusing spectral structures. The shaping of the antenna elements was aimed at providing efficiencies that are maximally smooth (Satyanarayana [114]), so that the smooth foregrounds retain their smoothness in detected spectra without confusing any embedded 21$\mathrm{cm}$ signal. The first SARAS experiment used a fat-dipole antenna [99]. Recognizing the advantages of monopole antennas over dipoles in their frequency

\footnotetext{
${ }^{10} \mathrm{http}: / /$ loco.lab.asu.edu/edges/

${ }^{11} \mathrm{http}: / /$ www.rri.res.in/DISTORTION/index.html
} 

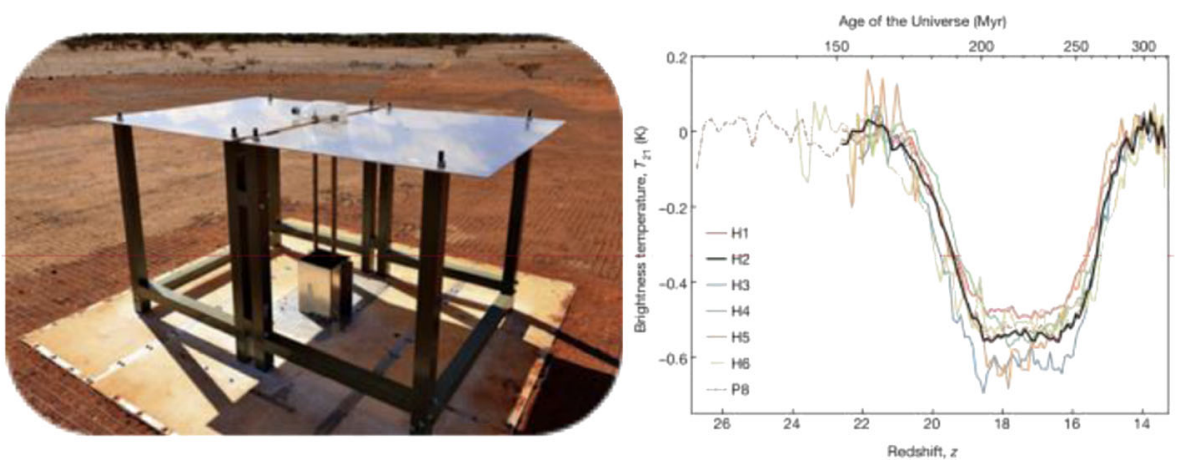

Fig. 4 The EDGES instrument (left) and its claimed global 21-cm signal detection (right; [15])

independence, the improved SARAS 2 devised a shaped monopole antenna [121]. Deployed in the radio-quiet Timbaktu Collective in Southern India, data in the 110-200 MHz band was examined for signatures of cosmological Reionization. The class of cosmological models (from the atlas of [27]) in which heating of primordial gas is inefficient-leading to deep absorption signals - together with rapid Reionization was rejected by the SARAS measurements [120, 122]. The progress in design for avoidance of spurious structures from the relatively intense foreground, and precision calibration methods that avoid receiver systematics, paved the way for a ground-based SARAS 3 that is optimized for the 50-

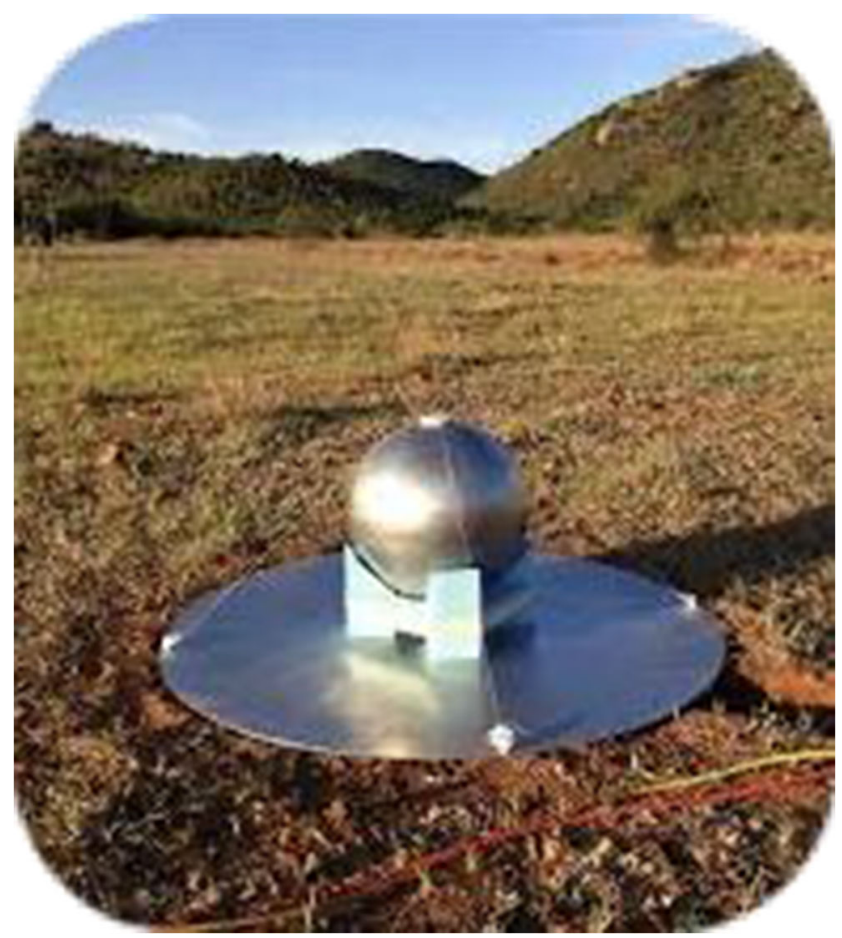

Fig. 5 The SARAS instrument 
$100 \mathrm{MHz}$ band. Additionally, recognizing the maturity in the ground experiments, the Indian space agency - ISRO - has provided the SARAS team pre-project funding for development of a lunar orbiter mission - PRATUSH http://www.rri. res.in/DISTORTION/pratush.html

\section{Current/planned space-based observatories}

Motivation for space-based $21-\mathrm{cm}$ signal observations Although current instruments (previous section) are slowly overcoming systematics related to the instrument (e.g. gain drifts, beam variations, etc.), the phase errors due to the ionosphere and still well beyond the ionospheric plasma frequency cut-off $(\sim 5-10 \mathrm{MHz})$, the extremely bright (extra)Galactic foregrounds (hundred to thousands of Kelvin) that outshine the $21-\mathrm{cm}$ signal (few to tens of $\mathrm{mK}$ ), and man-made interference, each of these effects become increasingly stronger toward lower frequencies. Even in the deserts of the Karoo desert of South Africa and Western Australia, one cannot escape RFI (e.g. from satellites, airplanes, etc.), and below 50 MHz the ionosphere becomes too turbulent to reach thermal-noise limited results. Even the slightest error of order $\sim 10^{-(4-6)}$ in the instrument or ionospheric corrections will be extremely detrimental for any high-redshift 21-cm experiment. Each of these potential show-stoppers motivate a space-based radio array.

- No ionosphere: Removing the detrimental impact of the ionosphere on the measurement electric field at each radio receiver, leading to both phase and amplitude errors, allows one to push observations to much higher redshifts, $\mathrm{z}>>25$, potentially even below the ionospheric cut-off to the early stages of the Dark Ages $(\mathrm{z} \sim 200, v \sim 7 \mathrm{MHz})$. Although the lunar surface might be mildly ionized, this will not be the case in lunar orbit, in lunar L2, nor in outer space. On Earth, the ionosphere requires second to minute time-scale calibration at $\sim 100 \mathrm{MHz}$, and even faster at lower frequencies, which will be unfeasible for Dark Ages 21-cm observations. Moreover, on such short timescales the instrumental signal-to-noise is not sufficient to solve for all unknowns of the ionosphere, effectively leading to speckle noise (e.g. [130, 131]).

- Eliminate RFI: Interference signals limit the frequencies observable from Earth (some fully excluded such as in the FM band, DABs, etc.; see e.g. [91, 92]). RFI can also occur as very low level, currently not seen in the data, and hence can be a potential showstopper for deep Dark Ages 21-cm signal observations, requiring dynamic ranges of $\sim 10^{8}$ in the data $(80 \mathrm{~dB})$. In space, e.g. on the far-side of the Moon, in lunar orbit (Earth/Sun shadow zone), or in the distant Earth-Sun L2 point, RFI from either can be largely eliminated.

- Stable Instrument: In space the environment of the instrument is shielded from weather, and temperature variations can be controlled better or happen slowly. This leads to a much more stable and controlled instrument environment, allowing for a slower calibration duty cycle (thus higher $\mathrm{S} / \mathrm{N}$ ), unlike on the ground where humidity and large (day-night) temperature variations continuously impact the performance of the instrument over the duration of the experiments which can be 
many years of observations. To correct for these errors, careful direction dependent and full-Stokes calibration is needed (e.g. [137]).

Each of these have motivated the development of a wide range of space-based 21-cm signal experiments, some focussing on the global 21-cm signal (sky-averaged; in line with e.g. EDGES and SARAS) via well-designed single receiver instruments (di/ tripoles), and others focussed on the spatial fluctuations of the 21-cm signal (in line with e.g. LOFAR, MWA, etc.). Below we briefly summarise these ongoing efforts (some realized, some in planning phase):

Dapper" "Burns et al. [21] proposed a SmallSat low-frequency experiment called the Dark Ages Polarimeter PathfindER (DAPPER) to fly in conjunction with NASA's accelerated lunar exploration program. DAPPER is proposed to observe at frequencies 17-38 MHz (z 83-36). It will measure the amplitude of the $21-\mathrm{cm}$ spectrum to a level that will distinguish between the standard cosmological model and models with additional cooling derived from current EDGES results. DAPPER's science instrument consists of dual orthogonal dipole antennas and a tone-injection spectrometer/ polarimeter based on high heritage components from the Parker Solar Probe/FIELDS, THEMIS, and the Van Allen Probes. DAPPER will be deployed from the vicinity of NASA's Lunar Gateway or in cis-lunar space and descend to a 50ロ125 km lunar orbit using a deep-space spacecraft bus that has both high impulse and high delta-V. This orbit will facilitate the collection of 4615 hours of radio-quiet data over a 26.4 month lifetime. DAPPER will search for divergences from the standard model that will indicate new physics such as heating or cooling produced by dark matter. The Cosmic Dawn trough in the redshifted 21-cm spectrum is affected by the complex astrophysical history of the first luminous objects. Another trough is expected during the Dark Ages, prior to the formation of the first stars and thus determined entirely by cosmological phenomena (including dark matter). DAPPER will observe this pristine epoch (17-38 $\mathrm{MHz} ; \mathrm{z} \sim 83-36$ ), and will measure the amplitude of the 21-cm spectrum to the level required to distinguish the the standard cosmological model from that of additional cooling at >5-sigma. In addition to dark matter properties such as annihilation, decay, temperature, and interactions, the low-frequency background radiation level can significantly modify this trough. Hence, this observation constitutes a powerful, clean probe of exotic physics in the Dark Ages. A second objective for DAPPER will be to verify the recent EDGES results for Cosmic Dawn, in the uncontaminated environment above the lunar farside, with sparse frequency sampling from 55-107 MHz (z 25-12)."

FareSide" "FARSIDE (Farside Array for Radio Science Investigations of the Dark ages and Exoplanets) is a Probe-class concept [22] to place a low radio frequency interferometric array on the farside of the Moon. A NASA-funded design study, focused on the instrument, a deployment rover, the lander and base station, delivered an architecture broadly consistent with the requirements for a Probe mission (about $\$ 1.3$ billion). This notional architecture consists of 128 dual polarisation antennas

\footnotetext{
$12 \mathrm{https} / / /$ www.colorado.edu/ness/dark-ages-polarimeter-pathfinder-dapper; Text largely taken and adapted from Burns et al. [21]

${ }^{13}$ Text largely taken and adapted from Burns et al. [22]
} 
deployed across a $10 \mathrm{~km}$ area by a rover, and tethered to a base station for central processing, power and data transmission to the Lunar Gateway. FARSIDE would provide the capability to image the entire sky each minute in 1400 channels spanning frequencies from $100 \mathrm{kHz}$ to $40 \mathrm{MHz}$, extending down two orders of magnitude below bands accessible to ground-based radio astronomy. The lunar farside can simultaneously provide isolation from terrestrial radio frequency interference, auroral kilometric radiation, and plasma noise from the solar wind. It is thus the only location within the inner solar system from which sky noise limited observations can be carried out at sub$\mathrm{MHz}$ frequencies. This would enable near-continuous monitoring of the nearest stellar systems in the search for the radio signatures of coronal mass ejections and energetic particle events, and would also detect the magnetospheres for the nearest candidate habitable exoplanets. Simultaneously, FARSIDE would be used to characterize similar activity in our own solar system, from the Sun to the outer planets, including the hypothetical Planet Nine. Through precision calibration via an orbiting beacon, and exquisite foreground characterization, FARSIDE would also measure the Dark Ages global 21-cm signal at redshifts $\mathrm{z} \sim 50-100$."

DSL $^{14}$ In the Discovering the Sky at the Longest Wavelengths (DSL) mission concept [26], a constellation of micro-satellites circling the Moon on nearly-identical orbits, form a linear array while making interferometric observations of the sky. The mission can map the sky below $30 \mathrm{MHz}$, which is still largely unknown. Although the sensitivity of such an array is insufficient to detect the fluctuating 21-cm signal from the Dark Ages, it could make a useful first step by mapping out the foreground, and also probe the Dark Ages through a precision global spectrum measurement using a single antenna. The observations will also be useful in a number of other fields, such as the study of Sun and planets, the interstellar medium, extragalactic radio sources, etc. In the DSL concept, a larger "mother" satellite leads or trails 5 to 8 smaller daughter satellites. The daughter satellites take the radio observation, and pass the data to the mother satellite using microwave link higher frequency bands. The microwave also serves for clock synchronization and distance ranging. The mother handles the interferometry computation and communication with the Earth. The relative position of the daughters are determined from the ranging information and angular measurement of the star sensor cameras. A lunar orbit mission would be simpler and less expensive than a lunar surface mission of similar capacity: it does not need to land on the Moon, thus it saves the required weight of the landing system. Furthermore, the low lunar orbit period is only a little more than two hours, there is no need to deal with long lunar nights and conventional solar power suffices. The data can be transmitted back to Earth during the time when the satellites are at the near side part of the orbit, therefore there is no need to have relay satellites. The whole DSL constellation can be launched with a single CZ-2C rocket. The DSL project is now undergoing a background prototype study.

NCLE $^{15}$ On May 21st, 2018, a Chinese Long March 3 rocket was launched from the Xichang launch base which brought the Queqiao relay satellite, as part of the Chang'e 4 mission, to space. The relay satellite is now over two and half years

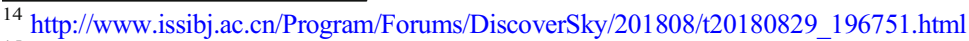

${ }^{15}$ https://www.ru.nl/astrophysics/radboud-radio-lab/projects/netherlands-china-low-frequency-explorer-ncle/
} 
behind the Moon in the Earth-Moon second Lagrange point, and was used during the historical landing on the Lunar far-side in January 2019. However, the Queqiao satellite also carries a low-frequency radio instrument payload, the NetherlandsChina Low frequency Explorer (NCLE). NCLE is designed, built and tested by a Dutch consortium comprised of the Radboud University (PI Falcke, Dept, PI Klein Wolt), ASTRON (Co-PI Boonstra) and ISIS (Delft), in close collaboration with the National Astronomical Observatories of the Chinese Academy of Sciences (NAOC, Co-PI Ping). The instrument has three main components. The three antenna units are each five-metre-long carbon-fibre monopoles (Fig. 6) that can be switched into dipole mode. The analogue system is sky noise limited in the 2-50 MHz regime, but the system is sensitive in the $80 \mathrm{kHz}$ to $80 \mathrm{MHz}$ regime, and in order to effectively deal with RFI and EMI peaks this range is split in 3 bands (16 channels 7.5$0.9 \mathrm{kHz}):<3 \mathrm{MHz}, 1-60 \mathrm{MHz}$ and $60-80 \mathrm{MHz}$. There are six $1 \mathrm{MHz}$ high-pass analogue modes and $10 \mathrm{MHz}$ high-pass modes, and two $3 \mathrm{MHz}$ low-pass analogue modes. The digital system has a 14-bit ADC (120 MHz), with full polarisation, $250 \mathrm{~GB}$ of on-board storage capacity and in total 39 digital modes. There are in total nine different science cases, ranging from solar bursts and Earth RFI to Dark Ages and Cosmic Dawn science, that can be addressed by choosing the right combination

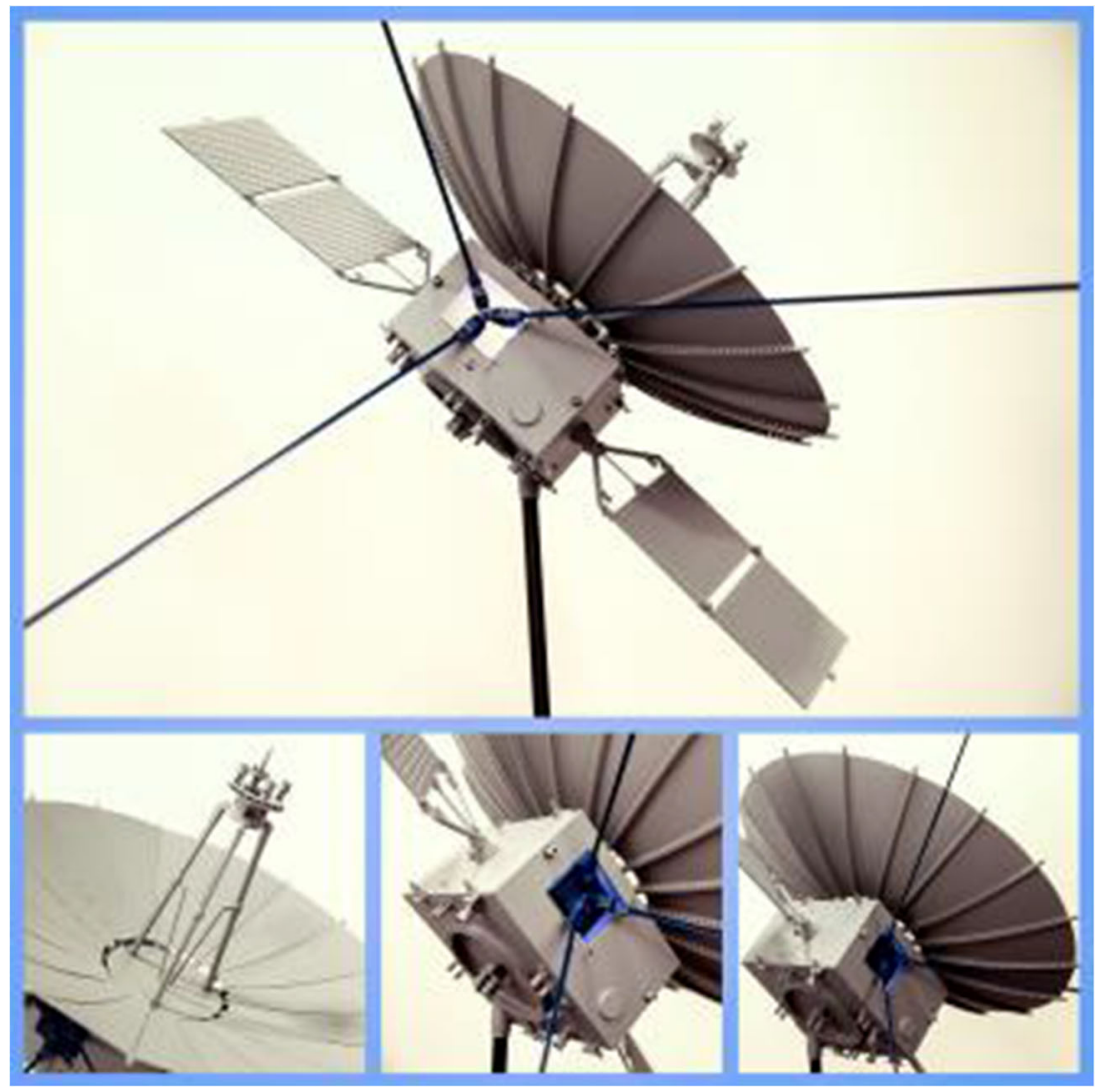

Fig. 6 Rendered view of NCLE, the tripole attached to the Chang-e'4 relay satellite 
of analogue and digital modes which will set the frequency regime of interest, the spectral and time resolution, and the total observing time. While NCLE opens up a virtually unexplored frequency domain for a wealth of interesting science cases, the RFI characterization is one of the most important technical objectives. NCLE will characterize the RFI and general noise environment behind the Moon, a site that has been identified as being ideal for future low frequency radio missions. The major science potential will be the attempt to detect the global Dark Ages and Cosmic Dawn signals, regarded as the "holy grail" of cosmology, but NCLE will also provide unique measurements of bright sources like the Sun and Jupiter, and attempt to create the most accurate (up to a few degrees) map of the sky at frequencies below $\sim 10 \mathrm{MHz}$. While being in space already since May 2018, the NCLE instrument was only allowed to start the commissioning after the Chinese lander mission on the lunar far-side had progressed significantly. Currently, the Dutch NCLE team is analyzing the first test data (Fig. 7) from the instrument and has started working on the commissioning. The first data shows that the NCLE hardware is still in a perfect condition, and that on top of the broad band variations caused by the instrument response there are sharp noise peaks most likely related to the onboard electronics of the Queqiao satellite. Note that these data were taken during a one-minute integration end-to-end test that was concluded successfully, and that during that time the antenna units are not deployed. After careful calibration of the instrument response as a function of its orbit around the Earth, the antennas have been deployed in November 2019 in a step-wise approach. Visual conformation via an onboard camera on the spacecraft of the deployment process shows the three individual antenna elements, however, the telemetry during the deployment process seems to suggest that the deployment was only a partial

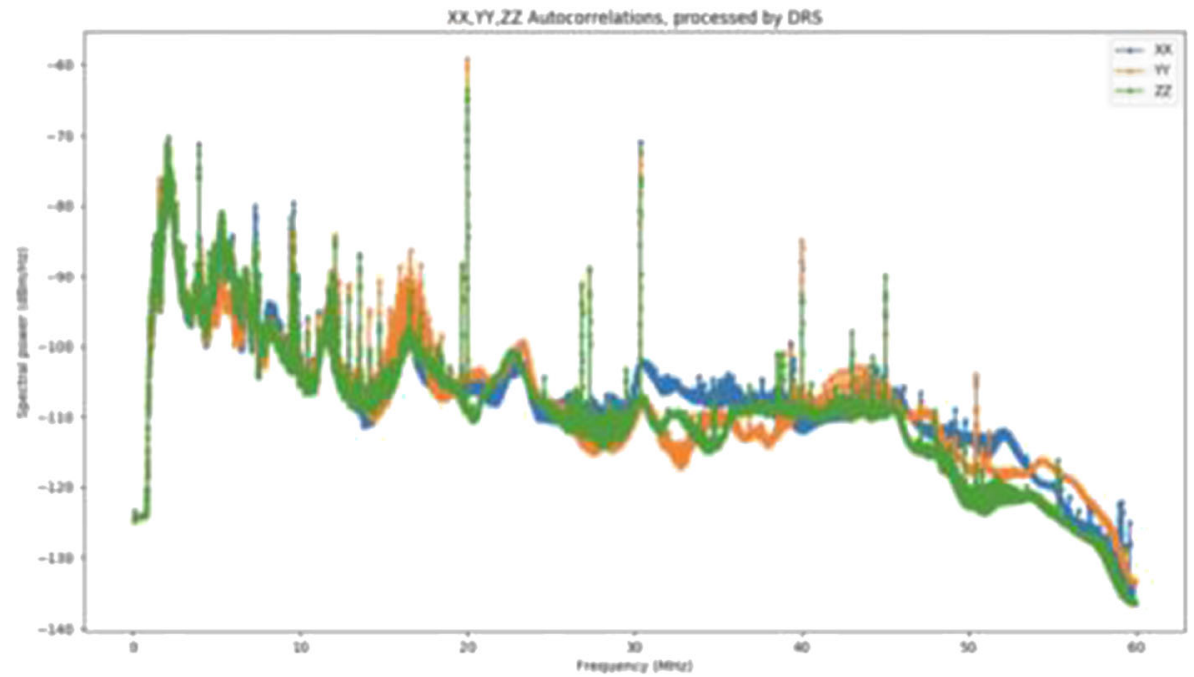

Fig. 7 First data taken during an end-to-end system test, without deployed antennas., in January 2019. The different colours represent the autocorrelations from the three antenna units. On top of the broad-band variations, which represent the variations in the response, there are clear sharp noise peaks most likely coming from electronic systems on the spacecraft 
success. One of the antennas is completely deployed, but the other two elements are most likely only $50 \%$ deployed. After the commissioning and calibration phase and the first science observations, which is still ongoing and can be done in the current antenna status, attempts will be made to fully deploy all threeantennas. Note that due to the combination of a technical issue and the Covid-19 situation, the transfer of data from the spacecraft to the ground has been delayed. More recently a temporal solution for the technical issues has been found, and the commissioning and calibration of the NCLE instrument is expected to be completed towards the second half of 2021 .

\section{CoDEX - Space/lunar-based mission design considerations}

Having discussed ongoing ground and space-based 21-cm instruments, we now consider some generic mission design considerations that enable one to observe the 21-cm signal from the Dark Ages $(z>25)$. These requirements are sufficiently stringent and inclusive that they also allow the lower-redshift 21-cm signal from the Cosmic Dawn $(\mathrm{z}<30)$ to be observed. To set the scene, but not to be complete in our analysis, we present general requirements for two representative redshifts (see Fig. 1). We generically refer to such a mission concept as CoDEX, the Cosmic-dawn Dark-ages EXplorer. CoDEX should be able to.

A. measure the 21-cm signal power spectra at the end of the Dark Ages $(z \sim 30)$ with $\mathrm{S} / N>10$ and image the 21-cm signal (tomography) with $\mathrm{S} / \mathrm{N}>10$, over at least one dex in k-modes.

B. measure the 21-cm signal power spectra at the peak of the Dark Ages $(\mathrm{z} \sim 50)$ with $\mathrm{S} / N>10$ and image the 21-cm signal (tomography) with $\mathrm{S} / \mathrm{N}>10$, over at least one dex in k-modes.

Below we calculate the $\mathrm{S} / \mathrm{N}$ ratios of CoDEX assuming a 5-year mission lifetime, Bandwidth $=10 \mathrm{MHz}$, and full-sky imaging.

\subsection{Basic instrument requirements of CODEX}

The most basic technical requirements of CoDEX are tied to the extreme surface brightness sensitivity required to reach the faint (mK-level) $21-\mathrm{cm}$ signal levels in the presence of very bright polarised foregrounds. This effectively translates into stringent requirements on the.

(1) collecting area $\left(\mathrm{A}_{\text {coll }}\right)$ - the total integrated effective area inside the core of the array,

(2) filling factor $\left(\mathrm{f}=\mathrm{A}_{\text {coll }} / \mathrm{A}_{\text {core }}\right)$, inside the core of the array, and

(3) field of view $\Omega=\lambda^{2} / \mathrm{A}_{\text {eff, }}$ where $\mathrm{A}_{\text {eff }}$ is the effective collecting area per receiver (e.g. dipole) and $\lambda$ the wavelength at which one observes.

We refer to Koopmans et al. [67] and Mellema et al. [79] for details on the error-budget of the 21-cm signal power spectrum scales with these three specifications. To calculate 
Table 1 CoDEX expected 21-cm signal $\mathrm{S} / \mathrm{N}$ ratios for a 5 year mission lifetime assuming full-sky imaging. Image cubes have a depth of $10 \mathrm{MHz}$, centred on these redshifts. For the $\mathrm{S} / \mathrm{N}$ calculation we assume the fiducial model as predicted from $\Lambda$ CDM. The orange coloured boxes correspond to Fig. 8 . The green boxes reach our minimum power-spectrum requirements of $\mathrm{S} / \mathrm{N}>10$ over one dex in k-modes and the orange and blue boxes exceed these (either in $\mathrm{S} / \mathrm{N}$ or k-mode range) for both power spectrum measurements and tomography.

\begin{tabular}{|c|c|c|c|c|}
\hline CoDEX Mission & $\begin{array}{l}\text { Dark Ages z=30, } \\
\text { Power Spectra }\end{array}$ & $\begin{array}{l}\text { Dark Ages z=30, } \\
\text { Tomography }\end{array}$ & $\begin{array}{l}\text { Dark Ages z=50, } \\
\text { Power Spectra }\end{array}$ & $\begin{array}{l}\text { Dark Ages } \mathrm{z}=50 \text {, } \\
\text { Tomography }\end{array}$ \\
\hline $\begin{array}{l}\text { CoDEX } \\
\left(1 \mathrm{~km}^{2}\right) \\
\text { M-class }\end{array}$ & $\begin{array}{l}\mathrm{S} / \mathrm{N} \sim 10 \text { for } \\
\mathrm{k} \sim 0.01-0.1\end{array}$ & $\begin{array}{l}\mathrm{S} / \mathrm{N} \sim 5 \text { for } \\
\mathrm{k}=0.01\end{array}$ & $\mathrm{~S} / \mathrm{N}<1$ & $\mathrm{~S} / \mathrm{N}<1$ \\
\hline $\begin{array}{l}\text { CoDEX } \\
\left(10 \mathrm{~km}^{2}\right) \\
\text { L-class }\end{array}$ & $\begin{array}{l}\mathrm{S} / \mathrm{N} \sim 10-100 \text { for } \mathrm{k} \sim 0.01- \\
1.0\end{array}$ & $\begin{array}{l}\mathrm{S} / \mathrm{N} \sim 10-100 \text { for } \\
\mathrm{k} \sim 0.01-0.1\end{array}$ & $\begin{array}{l}\mathrm{S} / \mathrm{N}>10 \text { for } \\
\mathrm{k} \sim 0.01-1\end{array}$ & $\begin{array}{l}\mathrm{S} / \mathrm{N}>10 \text { for } \\
\mathrm{k} \sim 0.01\end{array}$ \\
\hline $\begin{array}{l}\text { CoDEX } \\
\left(100 \mathrm{~km}^{2}\right) \\
\text { L-class }\end{array}$ & $\begin{array}{l}\mathrm{S} / \mathrm{N} \sim 100-1000 \text { for } \mathrm{k} \sim 0.01- \\
1.0\end{array}$ & $\begin{array}{l}\mathrm{S} / \mathrm{N} \sim 10-1000 \text { for } \\
\mathrm{k} \sim 0.01-0.4\end{array}$ & $\begin{array}{l}\mathrm{S} / \mathrm{N}>100 \text { for } \\
\mathrm{k} \sim 0.01-1\end{array}$ & $\begin{array}{l}\mathrm{S} / \mathrm{N} \sim 10-100 \text { for } \\
\mathrm{k} \sim 0.01-0.1\end{array}$ \\
\hline
\end{tabular}

the $\mathrm{S} / \mathrm{N}$, we use the more detailed formalism of McQuinn et al. [74] and assume the Galactic foregrounds also used for SKA models. The results are listed in Table.1.

These results shows that a $\mathrm{S} / \mathrm{N}>10$ can be reached over a very wide range of scales and tomography can be performed as well for $z=30$ if CoDEX has a collecting area of at least $10 \mathrm{~km}^{2}$, although some more modest targets (power-spectrum) can be reached at $\mathrm{z}=30$ with a collecting area of $1 \mathrm{~km}^{2}$. However, at least $100 \mathrm{~km}^{2}$ collecting area is needed for tomography at $\mathrm{z}=50$ with $\mathrm{S} / \mathrm{N}>10$ over $\mathrm{k} \sim 0.01-0.1 \mathrm{Mpc}^{-1}$, which ultimate goal of CoDEX.

In summary, we require:

- A collecting area of $\sim \mathbf{1 0} \mathbf{k m}^{2}$ to reach a $\mathrm{S} / \mathrm{N}>10$ at $\mathrm{k}=0.01-0.1 \mathrm{Mpc}^{-1}$ at $\mathbf{z}=\mathbf{3 0}$ within a 5-year mission lifetime, over a $10 \mathrm{MHz}$ bandwidth (i.e. redshift range), assuming all-sky imaging, and an array filling factor of unity.

- A collecting area of $\sim \mathbf{1 0 0} \mathbf{k m}^{2}$ to reach a $\mathrm{S} / \mathrm{N}>10$ at $\mathrm{k} \sim 0.01-0.1 \mathrm{Mpc}^{-1}$ at $\mathbf{z}=\mathbf{5 0}$ within a 5-year mission lifetime, over a $10 \mathrm{MHz}$ bandwidth (i.e. redshift range), assuming all-sky imaging, and an array filling factor of unity.

We conclude that to measure the 21-cm signal from the Dark Ages from $z \sim 30$ up to $z \sim 50$, any space-based low-frequency interferometer should have a lifespan of at least 5 years, a collecting area of $>1 \mathbf{k m}^{2}$ (M-mission scale) for first results, expandable to $100 \mathrm{~km}^{2}$ (L-mission scale), a filling factor of unity and all-sky field of view to reach the minimum $S / N>10$ for both power-spectrum and tomography over at least one dex. in spatial scale. The technical requirements of such a generic instrument (CoDEX) are summarised in Table 2.

\subsection{Mission concept considerations}

We now look in particular at a swarm of (cube)sats and/or connected receivers, either in Sun-Earth or lunar L2, in lunar orbit or on the surface of the Moon. Each option has 

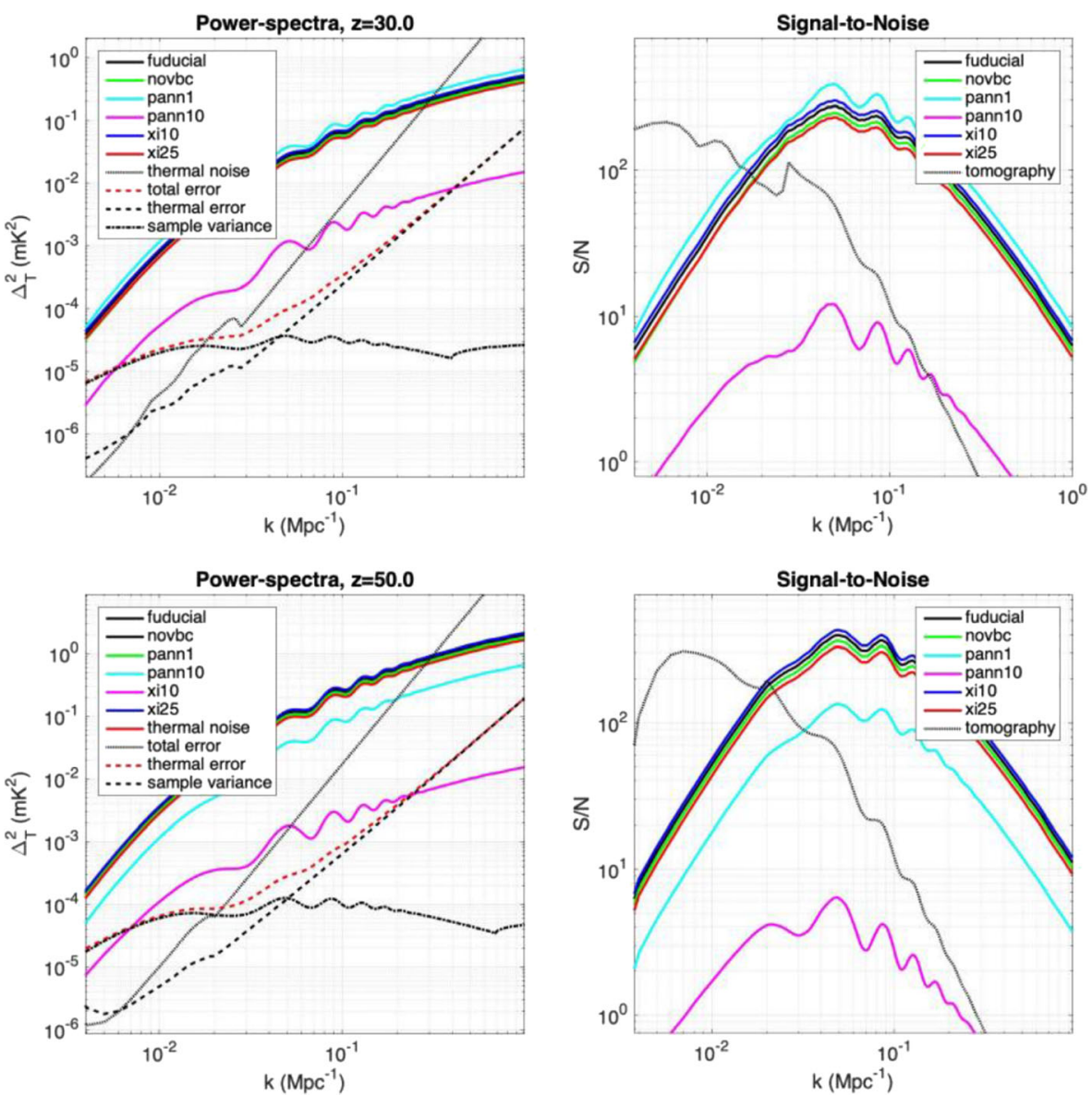

Fig. 8 Top Left: Simulated power spectra of the 21-cm line based on Yacine et al. ([136]); Xingang et al. ([134]) (top solid solid lines; the fiducial model is in black (the top four power spectra are offset by $10 \%$ for clarity) and the expected sensitivity of the DEX array (red dashed line) for $\mathrm{z}=30$ for a $10 \mathrm{~km} 2$ core and a 5year mission, using a BW =10 MHz. Top Right: Signal-to-noise achieved. Bottom Left/Right: Idem for $\mathrm{z}=50$ with a $100 \mathrm{~km} 2$ core. In both cases, the dotted line indicated the thermal-noise power-spectrum. In case the 21$\mathrm{cm}$ signal exceeds the noise power spectrum, direct imaging of those scales (tomography) can be done. We note that direct imaging on the largest scales is not affected by sample variance and can typically be done for scales larger than half a degree $(\mathrm{k} \sim 0.1)$

commonalities, requiring large collecting areas, a high filling factor, and a large field of view. We again refer to this generic concept as CoDEX, the Cosmic-dawn Dark-ages EXplorer.

\subsection{Free-space mission concept}

Based on previous designs for space-based, low-frequency, interferometry missions such as DARIS [29], SURO, HEIMDAL, DARE [20] and OLFAR [33], and most recently DEX (Klein [62]), a generic space-based mission concept could be as follows:

- The individual antennas are (semi)omni-directional dipoles or tripoles (active 
Table 2 CoDEX technical requirements

\begin{tabular}{|c|c|c|c|}
\hline \multicolumn{2}{|c|}{$\begin{array}{l}\mathrm{T} \text { e c h n i c a } 1 \\
\text { Requirements }\end{array}$} & \multirow{2}{*}{$\begin{array}{l}\text { Value / range } \\
1-100 \mathrm{MHz}\end{array}$} & \multirow{2}{*}{$\begin{array}{l}\text { Comments } \\
\text { Covers Dark Ages and Cosmic Dawn }\end{array}$} \\
\hline TR01 & $\begin{array}{l}\text { Frequency } \\
\text { range }\end{array}$ & & \\
\hline \multirow[t]{4}{*}{ TR02 } & Frequency & resolution & $\begin{array}{l}1 \mathrm{kHz}(\mathrm{RFI} / \mathrm{burst} \text { mode) } \\
10-100 \mathrm{kHz} \text { (Dark Ages and Cosmic Dawn science) }\end{array}$ \\
\hline & RFI & $\begin{array}{l}\text { excision/Limit bandwidth } \\
\text { smearing/Ancillary Science }\end{array}$ & \\
\hline & & Bandwidth & $\begin{array}{l}50 \mathrm{MHz} \text { (between } 1 \text { and } 100 \mathrm{MHz} \text { ) } \\
\text { tuneable }\end{array}$ \\
\hline & & $\begin{array}{l}\text { Covering } z=50-20 \text { in one go } \\
\text { and provide calibration } \\
\text { bandwidth }\end{array}$ & \\
\hline \multirow[t]{2}{*}{ TR03 } & Time & resolution & $50 \mathrm{~ms}$ (RFI/burst mode) \\
\hline & & & 10s (Dark Ages and Cosmic Dawn science) \\
\hline RFI & & $\begin{array}{l}\text { excision/Dark Ages and Cos- } \\
\text { mic Dawn science burst } \\
\text { mode/ Ancillary Science }\end{array}$ & \\
\hline TR04 & $\begin{array}{l}\text { Core area } \\
\text { of the } \\
\text { array }\end{array}$ & $A_{\text {core }}=1-10-100 \mathrm{~km}^{2}$ & With filling factor $\sim 1$ \\
\hline \multirow[t]{2}{*}{ TR05 } & Array & elements & $3 \times 10^{4-5-6}$ \\
\hline & & $\begin{array}{l}\text { Depends on optimal } \lambda \text { and } \\
\mathrm{A}_{\text {core; }} \text { most are in a } \\
\text { "fully-filled" core }\end{array}$ & \\
\hline \multirow[t]{2}{*}{ TR06 } & Number of & baselines & $10^{9-11-13}$ \\
\hline & $\begin{array}{l}\text { Possibly } \\
\text { FFT }\end{array}$ & correlation core & \\
\hline TR07 & $\begin{array}{l}\text { Baseline } \\
\text { lengths }\end{array}$ & $\begin{array}{l}<=10 \mathrm{~km} \text { (core) } \\
<=100 \mathrm{~km} \text { (outer) }\end{array}$ & $\begin{array}{l}\text { "Satellite" outer stations up to } 100 \mathrm{~km} \text {. Core } \\
\text { resolution }<10^{\prime} \text { and }<0.1 \text { ' on long baselines, at } \mathrm{z}=50 \\
\text { (better at lower } \mathrm{z} \text { ). }\end{array}$ \\
\hline TR08 & $\begin{array}{l}\text { Element } \\
\text { field of } \\
\text { view }\end{array}$ & $\begin{array}{l}4 \pi \text { sr with side-lobes for space } \\
\text { array, about } 2 \pi \text { sr for lunar } \\
\text { array }\end{array}$ & $\begin{array}{l}\text { "all-sky" coverage for a space array, one hemisphere } \\
\text { per snapshot for a lunar array }\end{array}$ \\
\hline
\end{tabular}

antennas), with their sensitivity optimized in the $1-80 \mathrm{MHz}$ regime over a bandwidth of $40 \mathrm{MHz}$. An array of core antennas can be placed on inflatable/foldable space structures or connectable nano-satellites, putting them in a (regular) grid ('core') to provide a high filing factor ( $f \sim 1$ ) optimally sensitive to the very faint 21 $\mathrm{cm}$ signals, surrounded by outer antennas (e.g. free-floating satellites) to enable the higher resolution imaging.

- The free-floating antennas are mounted on nano or small satellites that provide power (solar panels) and basic processing (RFI excision, FFT and averaging algorithms) and communication. Together with a mother-ship, possibly mounted on or closely located to the core, they provide data storage, processing, and communication with Earth. The constellation acts as a "swarm". 
- The core provides the sensitivity for observations of the Cosmic Dawn and Dark Ages, providing perfect uv coverage. The outer free-floating satellites/antenna provide superb imaging quality at higher angular resolutions. Due to the motion of the individual antennas, the baselines are constantly changing and complete coverage of the $(u, v, w)$ plane is achieved.

- The array should be placed in a RFI-quiet location, for instance at the Sun-Earth L2 point or in an Earth-leading or Earth-trailing orbit, or in lunar orbit (similar to DLS). This will minimize the need for RFI-mitigation techniques.

- Each individual antenna should be calibrated carefully and the noise pattern (EMC) should be characterized in detail. Stringent satellite EMC measures need to be implemented, but previous single element radio missions have shown that this is feasible.

- Data processing can be done at a central mothership which has dedicated data processing facilities, more power available (larger solar panels) and which has a large data rate available for data transfer. The individual antennas then have to transfer the data to the mothership and have to perform on-board processing to reduce the data volume; for the core receivers the data-transport will be easier if mounted near the central processor.

- Alternatively, data processing and transfer can be arranged by the array of antennas, i.e. they act as a swarm, performing the signal processing in a distributed fashion. This has the advantage that with the increase of the array the processing power is increased, but also requires each individual element to be provided with significant processing and communication capabilities.

- For the communication between core antennas and the mothership, RF links can be used, for the individual antennas and the mothership, optical communication can be used;

\subsection{Lunar-based mission concept}

The CoDEX lunar concept design on the one hand draws from the previous proposed lunar mission concepts, but on the other hand will be based on the experience and expertise gained with Earth-based low-frequency interferometers such as LOFAR (van [54]) and in the future SKA and HERA. In particular, the experience in the technical realization of a large collecting area radio array in (often) remote locations, calibration of the instruments, RFI mitigation techniques, and the handling and processing of large data volumes is essential for the development of CoDEX. In short, the CoDEX lunar interferometer design has the following characteristics:

- Interferometer array, consisting of about $3 \times 10^{4-5-6}$ individual antenna elements together ultimately realizing a staged $1-10-100 \mathrm{~km}^{2}$ collecting area on the lunar surface, sensitive in the $1-100 \mathrm{MHz}$ frequency regime.

- The individual antenna elements can consist of traditional dipole or tripole antennas (e.g. LRX, see Klein [61]) or be placed on thin metal sheets that are rolled out on the surface (ROLSS, see [68]).

- The location should be chosen carefully in order to provide temperature and gain stability as well as attenuation of RFI signals from Earth and solar activity. The preferred location is the lunar far-side but South- or North Pole locations using 
shielding from mountains are also options. Note that while the lunar far-side is preferred, it does require an additional orbiter for communication to the Earth.

- At a Lunar far-side location (similar to FareSide; see Section 6) the data processing and communication should be done from an orbiter or relay satellite (as for Change'4/NCLE): it provides more power from solar panels and has better communications with Earth available compared to stationary platforms on the lunar far-side.

- Each individual antenna should be calibrated carefully and the noise pattern (EMC) should be characterized in detail.

\subsection{Key technologies - Some general considerations}

Here we provide an overview of some of the key technologies that are required for the mission concept, and provide some thoughts on technologies that could help build a large interferometer in space or on the lunar surface:

\subsubsection{Nano-satellites and Swarm technologies}

The main design considerations for a free-floating astronomical low-frequency array in space relate to the physical characteristics of the interplanetary and interstellar medium. The system must consist of a high filling factor "core" plus a swarm of identical satellites spread over kilometric distances that will orbit far away from terrestrial radio frequency interference. To reduce weight one needs to miniaturize the electronics and perhaps use nano satellites with masses between 1 and $10 \mathrm{~kg}$. One approach is to use a swarm of satellites to establish a virtual telescope. In recent studies, such as DARIS [113] and FIRST [12] it is shown that with extrapolation of current signal processing and satellite technologies, a low frequency radio telescope in space could be feasible. DARIS has shown that a 9-satellite cluster, with a centralized system can be implemented in lunar orbit with today's technology (see also DSL above). Similar to the OLFAR project ([11]; Budianu et al. [19], [34, 111]), CoDEX needs scalable autonomous satellite flight units. To achieve sufficient spatial resolution, the minimum distances between the satellites must be more than $10 \mathrm{~km}$ and due to interstellar scattering this maximum baseline is limited to $100 \mathrm{~km}$, giving a resolution of 1 arc minute at $10 \mathrm{MHz}$. Each individual satellite will consist of deployable (e.g. tripole) antennas. As the satellites will be far away from Earth, communication to and from Earth will require diverse communication schemes, using all the individual satellites together.

\subsubsection{Inflatable space structures}

CoDEX requires a significant collecting area (staged in steps, e.g. 1-10-100 $\mathrm{km}^{2}$ ) and while radio antenna and receiver technologies are well developed (TRL levels of 6 and higher), bringing them into space or to the Moon is a costly and technologically challenging endeavour. In order to reduce the weight of the mechanisms required for the deployment and hence the costs of such a mission, the use of inflatable space structures is suggested. Another reason for using inflatable structures is the required filling factor in the core of the instrument. A swarm of antennas cannot easily obtain a 
filling factor of 1 without colliding and an inflatable structure can help to create the 1$10-100 \mathrm{~km}^{2}$ with $\mathrm{f}=1$ to starting doing interesting observations of the Dark Ages and Cosmic Dawn. The first two missions using large inflatable structures launched by NASA in 1960, Echo 1 and 2, were successfully used as communication reflectors. Interest in inflatable space structures was renewed which the successful deployment of the NASA Inflatable Antenna Experiment (IAE) from space shuttle mission STS-77 in 1996 [48]. In addition, several mission concepts were proposed, for instance MIT and JPL's mission to develop an inflatable antenna structure for cube sats [3]. For CoDEX the inflatable space structure technology can be adapted for a platform or deployment of the antenna system, comparable to the IAE concept. For instance, the scalable 1-10$100 \mathrm{~km}^{2}$ required for CoDEX can be obtained by forming an array of Echo2-like antennas, or placing them on flat foldable surfaces (e.g. "solar sail" material) surrounded by an inflatable structure to keep the area rigid. The latter would also allow a staged deployment to increase collecting area. Given the current development in the commercial heavy-launch space flight, the deployment and realization of CoDEX is becoming feasible in the near future from a technological and financial point-of-view.

\subsubsection{Additive manufacturing}

In order to minimize the number of launches and the payload required for construction of the radio-antenna array on the Moon or in space, the utilization of resources available on the Moon is highly desirable. It can dramatically reduce the overall project cost, provided that the resources present on the lunar surface are adequate and that the technology is available to transform these resources into the desired products and structures. The lunar surface consists of a mixture of dust, soil and broken rocks commonly referred to as regolith. The regolith is composed of various oxidecontaining minerals. Previous lunar missions have shown that the regolith composition varies in different regions of the Moon. The compositions in various regions can be found in, for example, [8], and [28]. The oxides of interest for in-situ construction of electrically conductive antennas are the metal-bearing ones (e.g. $\mathrm{TiO}_{2}, \mathrm{Al}_{2} \mathrm{O}_{3}, \mathrm{FeO}$ ). With the perspective of in-situ resource utilization for construction of the antenna array, Additive Manufacturing (AM) techniques can be of high interest, as they allow efficient material use and can be automated. The European Space Agency has developed and is currently investigating AM processes for the construction of hardware and infrastructure, to support the establishment of human settlements on the Moon and Mars (ESA workshop 2018, 2021 ${ }^{16}$ ). Such processes could potentially be applied - after required maturation and validation in a relevant environment - to build supporting infrastructure for the antenna array installations. Ongoing and planned activities at ESA also aim at studying the possibilities of using and recycling materials brought from Earth for the mission, in particular polymers. AM of polymers has been demonstrated on board the International Space Station, for relatively small parts. However, the use of such polymers to build the antenna arrays would require these materials to be made electrically conductive (e.g. by addition of conductive particles). In-orbit AM of such conductive composite materials has not been demonstrated yet. In addition, using polymer resources brought from Earth for the construction of the radio-telescope would

$\overline{16}$ https://atpi.eventsair.com/QuickEventWebsitePortal/advanced-manufacturing/home 
still require large payload mass. In order to build metallic antenna arrays using in-situ resources, the metallic elements would need to be extracted from the regolith. These metals could then be used as feedstock for AM processes. A range of AM processes exists for metallic products, using metallic feedstock in a powder or wire form. The adaption and validation of such processes for the lunar environment would need to be performed. Several techniques have been proposed for the extraction of metal resources from the regolith. These techniques are usually primarily intended to extract volatiles (oxygen, hydrogen or water) to support crewed missions. But they often yield metals and other solids as by-products. The as-extracted metallic by-products could be used, provided that they have the required electrical conductivity for the antenna application. Alternatively, the by-products would need to be beneficiated to the required purity. The processes that would turn the extracted metals into antenna arrays also need to be developed for the lunar environment. In addition, given the large collecting area required for the antenna array (up to $\sim 100 \mathrm{~km}^{2}$ ) the means of mining, sieving, and transporting the regolith to the desired manufacturing location need to be studied. Alternatively, a mobile AM system (e.g. mounted on a rover) needs to be developed. Energy transportation (e.g. power cables) from the energy harvesting sites to the manufacturing sites may also be required. Regarding the option of a space-based antenna array, no experience on in-orbit manufacturing of metallic products has been reported.

\subsubsection{Scalability}

The realization of a staged 1-10-100 $\mathrm{km}^{2}$ CoDEX interferometer in space or on the Moon is a significant technological challenge that should be approached in a step-wise fashion and depends on future developments in the areas of space transportation, lightweight inflatable antenna space structures, data communication and low-power-highperformance data processing and swarm technologies. The CoDEX concepts (both the space-based and the lunar-based concepts) are based on the same science case and have commonalities with respect to the data processing and operational modes, but also are equally flexible and scalable. In both cases individual elements can be added to the array which would increase the sensitivity and science output, and in some cases also the total data processing capabilities. Starting a $0.1 \mathrm{~km}^{2}$ CoDEX array, as a pathfinder mission, would also immediately provide valuable science output; not only would the CoDEX pathfinder open up the last unexplored frequency domain, but it would allow for the detection of the global Dark Ages signal as well address many of the secondary science cases (planetary radio emission, all sky survey etc). As elements are added to the CoDEX array, more science-domains will open.

\subsubsection{TRL levels}

In Table 3 we present an overview of the technologies introduced in the previous section that are required for a CoDEX mission. In this table we have indicated the current TRL levels, as well as the level expected to be reached at the end of the Ref. "Budianu 2015" is cited in the body but its bibliographic information is missing. Kindly provide its bibliographic information in the list. timeframe, and the critical developments that are required to be able to reach that level. The TRL level are indications and 
Table 3 TRL level for some of the CoDEX technologies

\begin{tabular}{|c|c|c|c|}
\hline Technologies & $\begin{array}{l}\text { Current } \\
\text { TRL }\end{array}$ & $\begin{array}{l}\text { Expected } \\
\text { TRL 2020- } \\
2030\end{array}$ & Expected critical developments \\
\hline $\begin{array}{l}\text { Radio Antenna (lightweight, } \\
\text { foldable, inflatable) }\end{array}$ & 5 & $6-7$ & $\begin{array}{l}\text { Small, foldable lightweight structures are being } \\
\text { designed in the OLFAR and ROLSS project that } \\
\text { should fit a nano-satellite. Current TRL } \\
\text { increasing activity is the NCLE antenna + } \\
\text { deployment system that is being designed for the } \\
\text { Chang'e } 4 \text { mission (launched in 2018, PI Falcke) }\end{array}$ \\
\hline $\begin{array}{l}\text { Radio Receiver (low-power, } \\
\text { high processing, } 200 \mathrm{MHz} \\
\text { receivers) }\end{array}$ & $5-6$ & $6-7$ & $\begin{array}{l}\text { Prototype radio receivers are expected to be tested } \\
\text { in rocket flights in the near future, and similar } \\
\text { systems will be tested in space environments } \\
\text { (e.g. ISS). Further heritage is gained from } \\
\text { ground-based low-frequency instruments such as } \\
\text { LOFAR, SKA, MWA, LWA, and space-based } \\
\text { instruments such as LRO. Current TRL increas- } \\
\text { ing activity is the NCLE receiver system that is } \\
\text { being designed for the Chang'e } 4 \text { mission } \\
\text { (launched in 2018, PI Falcke) }\end{array}$ \\
\hline Digital processing system & $4-5$ & $6-7$ & $\begin{array}{l}\text { Development of power-saving and smart algo- } \\
\text { rithms to process large quantities of data with } \\
\text { significantly less power are currently ongoing in } \\
\text { many Big-Data Science projects (CERN, ITER, } \\
\text { LOFAR, SKA) }\end{array}$ \\
\hline Optical communication & $6-8$ & $7-9$ & $\begin{array}{l}\text { Optical communication and nano-photonics are } \\
\text { expected to be employed in the space industry } \\
\text { (telecom) and have been tested since the } 1970 \text { s } \\
\text { (e.g. SILEX on ESA Artemis) }\end{array}$ \\
\hline Swarm Technologies & $3-4$ & $5-6$ & $\begin{array}{l}\text { OLFAR, inter-satellite communication, satellite } \\
\text { control }\end{array}$ \\
\hline Thin-film solar panels & $4-5$ & $7-8$ & $\begin{array}{l}\text { Currently thin-film solar panels are considered for } \\
\text { future missions with expected launch dates be- } \\
\text { fore Athena and LISA. }\end{array}$ \\
\hline $\begin{array}{l}\text { Radio Frequency } \\
\text { interferometry }\end{array}$ & $7-9$ & $7-9$ & $\begin{array}{l}\text { Based on space-ground interferometers (HALCA } \\
\text { and RadioAstron), and there's been some crude } \\
\text { time-difference-of-arrival measurements } \\
\text { (interferometric-like) using the THEMIS or } \\
\text { Cluster spacecraft. }\end{array}$ \\
\hline Inflatable space structures & $7-8$ & $8-9$ & $\begin{array}{l}\text { NASA Echo1 and } 2 \text { missions, NASA inflatable } \\
\text { antenna experiment (IAE, 1996) }\end{array}$ \\
\hline Additive manufacturing & $4-5$ & $7-8$ & $\begin{array}{l}\text { Energy requirement for the manufacturing process, } \\
\text { extracting process (mining) from regolith, pro- } \\
\text { duction of conductive materials, impact of } \\
\text { vacuum, reduced gravity, the presence of abra- } \\
\text { sive and electrically charged regolith particles, as } \\
\text { well as the influence of temperature variations } \\
\text { on the lunar surface on the sintering process }\end{array}$ \\
\hline
\end{tabular}

are based on our observations and some discussions with experts. However, it is important to realize that some of the critical developments are happening now already, independently of the CoDEX initiative. We believe that CoDEX can benefit from these 
developments, but moreover could provide a very interesting (additional) science case to support them.

\section{Short Summary \& Conclusions}

In this White Paper, we have argued for the development of space-based technology that enables the detection of the 21-cm signal from neutral hydrogen at redshift up to $\mathrm{z}$ $\sim 50$, during the peak of the Dark Ages. This will be impossible from the ground, where the ionosphere is prohibitively volatile to enable such observations. Cosmology with the 21-cm signal of neutral hydrogen at high redshift has proven extremely difficult as demonstrated by many of the current ground-based instruments aiming for a first detection of the 21-cm signal from the Epoch of Reionization and late Cosmic Dawnbut it promises an entirely new avenue to study the infant Universe $(z>6)$, probing astrophysics of the first stars, galaxies, black holes, and intergalactic medium during the Cosmic Dawn $(z>10)$, and potentially unveiling entirely new physics during the Dark Ages $(z>30)$. The reason for these difficulties has been the unstable environment on Earth (weather, ionosphere, human-made RFI, etc) in which these ground-based instrument have to operate, the complexity of the instrument itself, and the extremely bright partly polarised (extra)Galactic foreground that are up to $10^{6}$ times brighter than the desired 21-cm signal.

The Dark Ages, however, ranging in redshift from $z \sim 30$ to $\sim 200$, is the absolute holy grail of 21-cm Cosmology. During this era, the Universe was pristine and linear perturbation theory holds. Currently well-understood physics should therefore be able to predict the $21-\mathrm{cm}$ signal from the Dark Ages with great precision, except if new physics manifests itself at the densities and scales probed at these high redshifts. Deviations from these standard-model predictions are direct evidence for physics beyond the standard model. Furthermore, the information contained in the $21-\mathrm{cm}$ signal contains many orders of magnitude more modes than incorporated in the CMB, which has already provided cosmologists with a wealth of new discoveries and led to two Nobel Prizes, or in future large-scale galaxy surveys such as those to be performed with the Rubin Observatory (previously known as LSST) or EUCLID.

Uncovering the 21-cm signal during the Dark Ages will arguably be one of the most daunting and difficult experiments ever undertaken, on par with the recent discoveries of gravitational waves and the Higgs boson. Developing this new frontier is well worth the effort and could uncover fundamental new insights into the origin of the Universe, as well as lead to the exploration of new physics.

\footnotetext{
Open Access This article is licensed under a Creative Commons Attribution 4.0 International License, which permits use, sharing, adaptation, distribution and reproduction in any medium or format, as long as you give appropriate credit to the original author(s) and the source, provide a link to the Creative Commons licence, and indicate if changes were made. The images or other third party material in this article are included in the article's Creative Commons licence, unless indicated otherwise in a credit line to the material. If material is not included in the article's Creative Commons licence and your intended use is not permitted by statutory regulation or exceeds the permitted use, you will need to obtain permission directly from the copyright holder. To view a copy of this licence, visit http://creativecommons.org/licenses/by/4.0/.
} 


\section{References}

1. Allison \& Dalgarno ApJ 158423 (1969)

2. Ansari et al., 2018, DoE Cosmic Visions Dark Energy Program, arXiv:1810.09572

3. Babuscia et al., 2014, Conference Paper in IEEE Aerospace Conference Proceedings. https://doi.org/ 10.1109/AERO.2014.7024296

4. Bernardi et al., AJ 799 (2015)

5. Bañados, et al.: Nature. 553, 473-476 (2018)

6. Barkana \& Loeb ApJ 626 1-11 (2005)

7. Barkana: Nature. 555, 71 (2018)

8. Basu, Molinaroli: Earth. Moon, and Planets. 85, 25-43 (1999)

9. Beardsley, et al.: ApJ. 833, 102 (2016)

10. Belostotski, I.E.E.E.: Transactions on Microwave Theory Techniques. 59, 178-187 (2011)

11. Bentum et al., 2009, 60th International Astronautical Congress, Daejeon, Republic of Korea, 12-16 October, 2009

12. Bergman et al., 2009, First explorer - an innovativelow-cost passive formation-flying system. In Proceedings of CEAS 2009 European air \& Space Conference, Manchester, UK, October 2009, Pages 1-14, Royal Aeronautical Society

13. Book, al al: Phys.Rev.Lett. 108, 211301 (2012)

14. Bowman, Rogers: Nature. 468, 796-798 (2010)

15. Bowman, et al.: Nature. 555, 67-70 (2018)

16. Bradley, et al.: ApJ. 874, 153 (2019)

17. Brandenberger, Cyr, Shi, 2019, JCAP 09 (2019) 009

18. Brorby et al., MNRAS 457 4081-4088 (2016)

19. Budianu, et al.: Acta astronautica. 107, 14-19 (2015)

20. Burns, et al.: AdSpR. 49, 433 (2012)

21. Burns et al. (2019a), American Astronomical Society Meeting \#234, id. 212.02. Bulletin of the American Astronomical Society, Vol. 51, No. 4

22. Burns et al. (2019b), Astro2020: Decadal Survey on Astronomy and Astrophysics, APC white papers, no. 178; Bulletin of the American Astronomical Society, Vol. 51, Issue 7, id. 178 (2019)

23. Carilli, et al.: Radio Science. Volume. 53, p845 (2018)

24. Cirelli, I., Panci: JCAP. 10, 009 (2009)

25. Chianese, et al.: Physics. Letters B. 790, 64-70 (2019)

26. Chen et al. (2019) https://arxiv.org/abs/1907.10853

27. Cohen et al., MNRAS 472 1915-1931 (2017)

28. Crawford 2015, Progress in Physical Geography: Earth and Environment, volume 39, issue 2

29. DARIS, 2010, Very Large Effective Receiving Antenna Aperture in Space, ESTEC/Contract 22108/08/ $\mathrm{NL} / \mathrm{ST}$

30. DeBoer, et al.: PASP. 129, 045001 (2017)

31. Draine \& Miralda-Escudé, ApJl 858 L10 (2018)

32. Eastwood et al., 2019, eprint arXiv:1906.08943

33. Engelen et al., 2010, 24th annual conference on small satellites, Utah, USA, August 9-12. 2010

34. Engelen, 2016 "Swarm Satellites", $\mathrm{PhD}$ thesis, TU Delft

35. Das, et al.: MNRAS. 469, 1166-1174 (2017)

36. Evoli, M., Ferrara: JCAP. 11, 024 (2014)

37. Ewall-Wice, et al.: MNRAS. 458, 2710-2724 (2016a)

38. Ewall-Wice, et al.: MNRAS. 460, 21 (2016b)

39. Ewall-Wice, et al.: ApJ. 868, 63 (2018)

40. Fan, et al.: The Astronomical Journal. 125(4), 1649-1659 (2003)

41. Fan, et al.: The Astronomical Journal. 132(1), 117-136 (2006)

42. Fialkov, et al.: MNRAS. 432, 2909-2916 (2013)

43. Fialkov, B., Cohen: PRL. 114, 101303 (2015)

44. Fialkov, B., Cohen: Phys. Rev. Lett. 121, 011101 (2018)

45. Fialkov, Barkana: MNRAS. 486, 1763 (2019)

46. Field, P.: IRE. 46, 240 (1958)

47. Field: ApJ. 129, 536 (1959)

48. Freeland et al., 1997, IAF-97, 1.3.01

49. Furlanetto: MNRAS. 370, 1867-1875 (2006) 
50. Furlanetto, O., Briggs: Physics Reports. 433, 181-301 (2006)

51. Fraser, et al.: Phys. Lett. B. 785, 159 (2018)

52. Gehlot, et al.: MNRAS. 488, 4271-4287 (2019)

53. Gehlot, et al.: MNRAS. 499, 4158-4173 (2020)

54. Haarlem, v., et al.: A\&A. 556, A2 (2013)

55. Haiman, R., Loeb: ApJ. 467, 522 (1996)

56. Hills, et al.: Nature. 564, E32-E34 (2018)

57. Hirata, et al.: PhRvD. 97, 103521 (2018)

58. Hogan, Rees: Monthly Notices of the Royal Astronomical Society. 188, 791 (1979)

59. Holzbauer, Furlanetto: MNRAS. 419, 718-731 (2012)

60. Hu, Weinreb: IEEE Transactions on Microwave Theory Techniques. 52, 1498-1507 (2004)

61. Wolt, K., et al.: P\&SS. 74, 167 (2012)

62. Klein Wolt et al., Dark Ages EXplorer, DEX, a White Paper for a Low Frequency Radio Interferometer Mission to Explore the Cosmological Dark Ages for the L2, L3 ESA Cosmic Vision Program, 2013, www.astro.ru.nl

63. Koh, Wise: MNRAS. 474, 3817-3824 (2018)

64. Kohn, et al.: ApJ. 823, 88 (2016)

65. Kohn, et al.: ApJ. 882, 58 (2018)

66. Kolopanis, et al.: AJ. 883, 133 (2019)

67. Koopmans et al., Advancing Astrophysics with the Square Kilometre Array (AASKA14) 1 (2015)

68. Lazio, et al.: Advances in. Space Research. 49, 1942-1957 (2011)

69. Lehmer, et al.: ApJ. 825, 7 (2016)

70. Lopez-Honorez, et al.: JCAP. 8, 004 (2016)

71. Madau, M., Rees: Astrophysical Journal. 475, 429 (1997)

72. Mason, et al.: MNRAS. 485, 3947-3969 (2019)

73. Masui, et al.: Phys.Rev.Lett. 118, 221301 (2017)

74. McQuinn, et al.: ApJ. 653, 815 (2006)

75. McQuinn, O'Leary: ApJ. 760, 3 (2012)

76. McQuinn: MNRAS. 426, 1349-1360 (2012)

77. Meerburg, et al.: JCAP. 1703, 03050 (2017)

78. Meerburg, et al.: JCAP. 1609, 09023 (2016)

79. Mellema, et al.: Experimental Astronomy. 36, 235-318 (2013)

80. Mesinger, F., Cen: MNRAS. 411, 955-972 (2011)

81. Mesinger, et al.: MNRAS. 459, 2342-2353 (2016)

82. Mineo, G., Sunyaev: MNRAS. 419, 2095-2115 (2012)

83. Monsalve, et al.: ApJ. 835, 49 (2017a)

84. Monsalve, et al:: ApJ. 847, 64 (2017b)

85. Monsalve, et al.: ApJ. 863, 11 (2018)

86. Monsalve, et al.: ApJ. 875, 67 (2019)

87. Mortlock, W.: Venemans, et al. Nature. 474(616), (2011)

88. Muñoz, Loeb: Nature. 557, 684-686 (2018a)

89. Muñoz, D., Loeb: PRL. 121, 1301 (2018b)

90. Muñoz: Phys. Rev. Lett. 123, 121201 (2019)

91. Offringa, et al.: A\&A. 549, A11 (2013)

92. Offringa, et al.: PASA. 32(e008), 13 (2015)

93. Pacucc, M., Mineo, Ferrara: MNRAS. 443, 678-686 (2014)

94. Pagica, et al.: MNRAS. 433, 639 (2013)

95. Park, et al.: MNRAS. 484, 933-949 (2019)

96. Parsons, et al.: AJ. 139, 1468-1480 (2010)

97. Parsons, et al.: ApJ. 753, -81 (2012)

98. Patil, et al.: The Astrophysical Journal. 838, 65 (2017)

99. Patra, S.: Raghunathan \& Shankar. Exp. Astron. 36(319), (2013)

100. Patra et al., 2018, Experimental Astronomy 45 (2), 177-199 
101. Pen, et al.: MNRAS. 399, 181-194 (2009)

102. Planck Collaboration XLVII: A\&A. 596, A108 (2016)

103. Planck Collaboration VI: A\&A. 641, A6 (2020)

104. Pober, et al.: ApJL. 768, L36 (2013)

105. Pober, et al.: ApJ. 782, 66 (2014)

106. Pospelov, et al.: Phys. Rev. Lett. 121, 31103 (2018)

107. Pritchard, Furlanetto: MNRAS. 376, 1680-1694 (2007)

108. Pritchard, Loeb: Phys.Rev. D. 78, 103511 (2008)

109. Pritchard, Loeb: Physical Review D. 82(2), 023006 (2010)

110. Pritchard, Loeb: Reports on Progress in Physics. 75, 086901 (2012)

111. Rajan, 2016, "Relative Space-Time Kinematics of an Anchorless Network", PhD thesis, TU Delft

112. Rogers \& Bowman Radio Science 47 RS0K06 (2012)

113. Saks et al., 2010, The 4S symposium, Madeira, Portugal, 31 may - 4 June 2010

114. Sathyanarayana Rao et al., ApJ 84033 (2017)

115. Schaerer: A\&A. 382, 28-42 (2002)

116. Schmidt, F., et al.: PhRvD. 89, 082507 (2014)

117. Scott \&. Rees, MNRAS 247, 510 (1990)

118. Senchyna et al., MNRAS 1840 (2019)

119. Sheere, C., et al.: PHRvD. 96, 063508 (2017)

120. Singh, et al.: ApJl. 845, -L12 (2017)

121. Singh, et al.: Experimental Astronomy. 45, 269-314 (2018a)

122. Singh, et al.: ApJ. 858, 54 (2018b)

123. Slatyer: PhRvD. 87, 3513 (2013)

124. Slatyer: PhRvD. 93, 3521 (2016a)

125. Slatyer: PhRvD. 93, 3527 (2016b)

126. Tashiro, K., Silk: Phys. Rev. D. 90, 083522 (2014)

127. Tingay, et al.: PASA. 30, -7 (2013)

128. Tumlinson, Shull: ApJl. 528, L65-L68 (2000)

129. Valdés, et al.: MNRAS. 429, 1705-1716 (2013)

130. Vedantham, et al.: MNRAS. 437, 1056-1069 (2014)

131. Vedantham, Koopmans: MNRAS. 458, 3099-3117 (2016)

132. Wayth, et al.: PASA. 35, E033 (2018). https://doi.org/10.1017/pasa.2018.37

133. Wouthuysen: Astronomical Journal. 57, 31 (1952)

134. Xingang, et al.: J. Cosmol. Astropart. Phys. 023, (2016)

135. Xu et al., ApJ 83384 (2016)

136. Yacine, et al.: Phys. Rev. D. 89(8) 083506 (2014)

137. Yatawatta, et al.: MNRAS. 475, 708-715 (2018)

138. Yoshida, O., Hernquist: Science. 321, 669 (2008)

139. Zaroubi, in The First Galaxies, Astrophysics and Space Science Library, Volume 396. ISBN 978-3642-32361-4. Springer-Verlag Berlin Heidelberg, 2013, p. 45

140. Zarka, et al. (2015) NenUFAR: Instrument description and science case. in 1-6 (IEEE), doi:https://doi. org/10.1109/ICATT.2015.7136773

141. Zheng et al., the Astrophysical Journal, Volume 832, Issue 2, article id. 190, 14 pp. (2016)

142. Zygelman: ApJ. 622, 1356-1362 (2005)

Publisher's note Springer Nature remains neutral with regard to jurisdictional claims in published maps and institutional affiliations. 


\section{Affiliations}

Léon V. E. Koopmans ${ }^{1} \cdot$ Rennan Barkana ${ }^{2} \cdot$ Mark Bentum $^{3,4} \cdot$ Gianni Bernardi $^{5,6}$. Albert-Jan Boonstra ${ }^{4} \cdot$ Judd Bowman $^{7}$ - Jack Burns ${ }^{8} \cdot$ Xuelei Chen $^{9}$ - Abhirup Datta $^{10}$. Heino Falcke ${ }^{11}$. Anastasia Fialkov ${ }^{12}$ - Bharat Gehlot ${ }^{7}$. Leonid

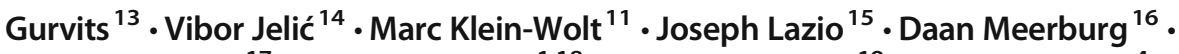
Garrelt Mellema $^{17}$. Florent Mertens ${ }^{1,18}$. Andrei Mesinger ${ }^{19}$ • André Offringa ${ }^{4}$. Jonathan Pritchard ${ }^{20}$. Benoit Semelin ${ }^{18}$ - Ravi Subrahmanyan ${ }^{21}$ - Joseph Silk $^{22,23}$ - Cathryn Trott ${ }^{24} \cdot$ Harish Vedantham ${ }^{4}$ - Licia Verde ${ }^{25}$ - Saleem Zaroubi $^{1,26} \cdot$ Philippe Zarka ${ }^{18}$

\section{Kapteyn Astronomical Institute, University of Groningen, Groningen, Netherlands}

Tel-Aviv University, Tel Aviv, Israel

3 Eindhoven University of Technology, Eindhoven, Netherlands

4 ASTRON, Dwingeloo, Netherlands

5 INAF, Bologna, Italy

6 SKAO-SA, Cape Town, South Africa

7 Arizona State University, Tempe, AZ, USA

8 University of Colorado Boulder, Boulder, CO, USA

$9 \quad$ NAOC, Beijing, China

10 IIT Indore, Indore, India

11 Radboud University, Nijmegen, Netherlands

12 IoA, University of Cambridge, Cambridge, UK

13 JIVE and Delft University of Technology, Delft, Netherlands

14 Institut Ruđer Bošković, Zagreb, Croatia

15 JPL, Caltech, Pasadena, CA, USA

VSI, University of Groningen, Groningen, Netherlands

Stockholm University, Stockholm, Sweden

8 Observatoire de Paris, Paris, France

19 Scuola Normale Superiore, Pisa, Italy

20 Imperial College, London, UK

21 Raman Research Institute, Bengaluru, India

22 Institut d'astrophysique de Paris, Paris, France

23 University of Oxford, Oxford, UK

24 Curtin University, Perth, Australia

25 Institute of Cosmological Sciences, Barcelona, Spain

26 Open University of Israel, Ra'anana, Israel 